\title{
Probabilistic Representation and Fall-Off of Bound States of Relativistic Schrödinger Operators with Spin 1/2
}

\author{
by \\ Fumio Hiroshima, Takashi ICHINose and József LőRINCZI
}

\begin{abstract}
A Feynman-Kac type formula for relativistic Schrödinger operators with unbounded vector potential and spin $1 / 2$ is given in terms of a three-component process consisting of a Brownian motion, a Poisson process and a subordinator. This formula is obtained for unbounded magnetic fields and magnetic fields with zeros. From this formula an energy comparison inequality is derived. Spatial decay of bound states is established separately for growing and for decaying potentials by using martingale methods.
\end{abstract}

2010 Mathematics Subject Classification: 47D07, 47D08, 60J75, 81Q10.

Keywords: relativistic Schrödinger operators, bound states, spatial decay, Feynman-Kac formulae, Poisson process, subordinate Brownian motion, martingales.

\section{$\S 1$. Introduction}

In the paper [HIL09] we constructed a Feynman-Kac formula for a generalized Schrödinger operator with spin of the form

$$
\Psi(h(a, \sigma))+V
$$

Here $V$ is a real-valued external potential, $\Psi$ is an arbitrary Bernstein function with $\Psi(0)=0$, and $h$ is a Schrödinger-type operator of the form

$$
h(a, \sigma)=\frac{1}{2}(\sigma \cdot(p-a))^{2},
$$

Communicated by T. Kumagai. Received April 19, 2012. Revised August 15, 2012.

F. Hiroshima: Faculty of Mathematics, Kyushu University, Fukuoka, 819-0395, Japan; e-mail: hiroshima@math.kyushu-u.ac.jp.

T. Ichinose: Department of Mathematics, Kanazawa University, Kanazawa, 920-11, Japan; e-mail: ichinose@kenroku.kanazawa-u.ac.jp

J. Lörinczi: School of Mathematics, Loughborough University, Loughborough LE11 3TU, United Kingdom;

e-mail: J.Lorinczi@lboro.ac.uk

(C) 2013 Research Institute for Mathematical Sciences, Kyoto University. All rights reserved. 
including a vector potential $a=\left(a_{1}, a_{2}, a_{3}\right)$ describing a magnetic field, and the Pauli matrices $\sigma=\left(\sigma_{1}, \sigma_{2}, \sigma_{3}\right)$ describing spin $1 / 2$. As we have shown, the Feynman-Kac representation of (1) involves three independent stochastic processes; a Brownian motion, a Poisson process and a subordinator. Moreover, spin $1 / 2$ was also extended to higher spins in [HIL09] (see also [ARS91]).

In this paper we consider a functional integral representation of the strongly continuous one-parameter semigroup generated by the relativistic Schrödinger operator with spin $1 / 2$ in three-dimensional space,

$$
\sqrt{(\sigma \cdot(p-a))^{2}+m^{2}}-m+V .
$$

Here $m$ is the mass of the relativistic particle, which we regard as a parameter; see [Car78] for standard Schrödinger operators, where $a=0$. This Hamilton operator is a special case of (1) obtained by choosing

$$
\Psi(u)=\sqrt{2 u+m^{2}}-m, \quad m \geq 0 .
$$

In this case we have the $\frac{1}{2}$-stable subordinator about which more details are known than about subordinators related to a general $\Psi$. Using this extra information, our main goal in this paper is to prove a Feynman-Kac type formula for (3) under weaker conditions than needed for general $\Psi$, and use it to derive the fall-off properties of bound states. In particular, in contrast to [HIL09] we can cover unbounded magnetic fields in Theorem 3.6 and magnetic fields with zeros in Theorem 3.8.

This paper is organized as follows. Section 2 is devoted to introducing the relativistic Schrödinger operator with spin $1 / 2$ as a self-adjoint operator on $\mathbb{C}^{2} \otimes$ $L^{2}\left(\mathbb{R}^{3}\right)$ and an equivalent unitary representation on $L^{2}\left(\mathbb{R}^{3} \times \mathbb{Z}_{2}\right)$. In Section 3.1 we reassess the results in [HIL09] and give a Feynman-Kac formula with bounded magnetic fields. In Section 3.2 we prove a Feynman-Kac formula for unbounded magnetic fields, and in Section 3.3 for magnetic fields having zeros. In Section 4 we derive the decay properties of bound states separately for growing and for decaying potentials by using martingale methods.

\section{§2. Relativistic Schrödinger operator with spin $1 / 2$}

\section{$\S 2.1$. Definitions}

We begin by defining the self-adjoint operator $h(a, \sigma)$ and $\sqrt{2 h(a, \sigma)+m^{2}}-m+V$ rigorously.

The spinless Schrödinger operator $h_{0}$ with vector potential $a$ and zero external potential is defined as a self-adjoint operator on $L^{2}\left(\mathbb{R}^{3}\right)$. Let $\mathrm{D}_{\mu}=p_{\mu}-a_{\mu}$, where 
$p_{\mu}=-i \partial_{x_{\mu}}$ is a momentum operator. Define a quadratic form $q$ by

$$
H^{1}\left(\mathbb{R}^{3}\right) \times H^{1}\left(\mathbb{R}^{3}\right) \ni(f, g) \mapsto q(f, g)=\frac{1}{2} \sum_{\mu=1}^{3}\left(\mathrm{D}_{\mu} f, \mathrm{D}_{\mu} g\right)
$$

where $H^{1}\left(\mathbb{R}^{3}\right)=\left\{f \in L^{2}\left(\mathbb{R}^{3}\right) \mid \mathrm{D}_{\mu} f \in L^{2}\left(\mathbb{R}^{3}\right), \mu=1,2,3\right\}$. If $a \in\left(L_{\text {loc }}^{2}\left(\mathbb{R}^{3}\right)\right)^{3}$, then the quadratic form $q$ is non-negative and closed, and hence there exists a unique self-adjoint operator $h_{0}$ satisfying $\left(h_{0} f, g\right)=q(f, g)$ for $f \in D\left(h_{0}\right)$ and $g \in H^{1}$, where $D\left(h_{0}\right)=\left\{f \in \mathrm{Q}(q) \mid q(f, \cdot) \in L^{2}\left(\mathbb{R}^{3}\right)^{\prime}\right\}$. Let $C_{0}^{\infty}\left(\mathbb{R}^{3}\right)=C_{0}^{\infty}$ be the set of infinitely differentiable functions with compact support on $\mathbb{R}^{3}$. It can be seen that $C_{0}^{\infty}$ is a form core for $h_{0}$ under the assumption $a \in\left(L_{\text {loc }}^{2}\left(\mathbb{R}^{3}\right)\right)^{3}$ (see [LS81]).

Next we introduce a magnetic field $b=\left(b_{1}, b_{2}, b_{3}\right)$. Physically it is given by $b=\nabla \times a$, but in this paper we consider the magnetic field $b$ independent of the vector potential $a$. We will use the following conditions on $a$.

Assumption 2.1 (Vector potential). The vector potential $a=\left(a_{1}, a_{2}, a_{3}\right)$ is a vector-valued function whose components $a_{\mu}, \mu=1,2,3$, are real-valued functions such that $a \in\left(L_{\text {loc }}^{2}\left(\mathbb{R}^{3}\right)\right)^{3}$ and $\nabla \cdot a \in L_{\text {loc }}^{1}\left(\mathbb{R}^{3}\right)$, where $\nabla \cdot a$ is understood in the distributional sense.

Assumption 2.2 (Magnetic field). $D(-\Delta) \subset D\left(b_{\mu}\right)$ and for $f \in D(-\Delta)$ the conditions $\left\|b_{\mu} f\right\| \leq \kappa_{\mu}\|-\Delta f\|+\kappa_{\mu}^{\prime}\|f\|, \mu=1,2,3$, and $\kappa_{1}+\kappa_{2}+\kappa_{3}<1$ are satisfied.

Finally we introduce the spin variables. Let $\sigma=\left(\sigma_{1}, \sigma_{2}, \sigma_{3}\right)$ be the $2 \times 2$ Pauli matrices given by

$$
\sigma_{1}=\left[\begin{array}{ll}
0 & 1 \\
1 & 0
\end{array}\right], \quad \sigma_{2}=\left[\begin{array}{cc}
0 & -i \\
i & 0
\end{array}\right], \quad \sigma_{3}=\left[\begin{array}{cc}
1 & 0 \\
0 & -1
\end{array}\right] .
$$

They satisfy the relations $\sigma_{\mu} \sigma_{\nu}+\sigma_{\nu} \sigma_{\mu}=2 \delta_{\mu \nu} 1$ and $\sigma_{\mu} \sigma_{\nu}=i \sum_{\lambda=1}^{3} \varepsilon^{\lambda \mu \nu} \sigma_{\lambda}$, where $\varepsilon^{\lambda \mu \nu}$ is the anti-symmetric Levi-Civita tensor with $\varepsilon^{123}=1$. Then it can be seen directly that

$$
\sigma \otimes b=\sum_{\mu=1}^{3} \sigma_{\mu} \otimes b_{\mu}=\left[\begin{array}{cc}
b_{3} & b_{1}-i b_{2} \\
b_{1}+b_{2} & -b_{3}
\end{array}\right] .
$$

Under Assumption 2.2, $\sigma \otimes b$ is relatively bounded with respect to $1 \otimes 2 h_{0}$, as an operator in $\mathbb{C}^{2} \otimes L^{2}\left(\mathbb{R}^{3}\right)$, with a relative bound strictly smaller than 1 ,

$$
\|(\sigma \otimes b) f\| \leq\left(\kappa_{1}+\kappa_{2}+\kappa_{3}\right)\left\|1 \otimes 2 h_{0} f\right\|+C\|f\|, \quad f \in \mathbb{C}^{2} \otimes D(h) .
$$


This follows through the diamagnetic inequality $\left|\left(f, e^{-t h_{0}} g\right)\right| \leq\left(|f|, e^{-t\left(-\frac{1}{2} \Delta\right)}|g|\right)$ under Assumption 2.1. Thus the self-adjoint operator

$$
h=1 \otimes h_{0}-\frac{1}{2} \sigma \otimes b
$$

in $\mathbb{C}^{2} \otimes L^{2}\left(\mathbb{R}^{3}\right)$ is bounded from below under Assumptions 2.1 and 2.2. We choose $m$ so as to guarantee that

$$
2 h+m^{2}=1 \otimes 2 h_{0}-\sigma \otimes b+m^{2} \geq 0 .
$$

Note that under a suitable condition $h$ is positive, and in this case we can take $m=0$. From now on we omit the tensor product $\otimes$ for notational convenience.

We now define the self-adjoint operator $H$.

Definition 2.3. Under Assumptions 2.1 and 2.2, $H$ is defined by the self-adjoint operator

$$
H=\sqrt{2 h+m^{2}}-m
$$

in $\mathbb{C}^{2} \otimes L^{2}\left(\mathbb{R}^{3}\right)$. Here the square root is taken through the spectral resolution of $2 h+m^{2}$.

A typical example is the operator $\sqrt{(\sigma \cdot(p-a))^{2}+m^{2}}-m$ such that $a \in$ $\left(L_{\text {loc }}^{4}\left(\mathbb{R}^{3}\right)\right)^{3}, \nabla \cdot a \in L_{\text {loc }}^{2}\left(\mathbb{R}^{3}\right)$ and $\nabla \times a \in\left(L_{\text {loc }}^{2}\left(\mathbb{R}^{3}\right)\right)^{3}$. In this case it is seen that

$$
(\sigma \cdot(p-a))^{2}=(p-a)^{2}+\sigma \cdot(\nabla \times a)
$$

on $1 \otimes C_{0}^{\infty}\left(\mathbb{R}^{3}\right)$

\section{§2.2. Spin variable}

In order to construct a functional integral representation of $\left(f, e^{-t(H+V)} g\right)$ we make a unitary transform of $H$ on $\mathbb{C}^{2} \otimes L^{2}\left(\mathbb{R}^{3}\right)$ to an operator on the space $L^{2}\left(\mathbb{R}^{3} \times \mathbb{Z}_{2}\right)$. This is a space of $L^{2}$-functions of $x \in \mathbb{R}^{3}$ and an additional two-valued spin variable $\theta \in \mathbb{Z}_{2}$, where

$$
\mathbb{Z}_{2}=\{-1,1\}
$$

We define the spin interaction $U$ on $L^{2}\left(\mathbb{R}^{3} \times \mathbb{Z}_{2}\right)$ by

$$
U: f(x, \theta) \mapsto U_{\mathrm{d}}(x, \theta) f(x, \theta)+U_{\mathrm{od}}(x,-\theta) f(x,-\theta)
$$

where $(x, \theta) \in \mathbb{R}^{3} \times \mathbb{Z}_{2}$,

$$
U_{\mathrm{d}}(x, \theta)=-\frac{1}{2} \theta b_{3}(x)
$$


is the diagonal component, and

$$
U_{\text {od }}(x,-\theta)=-\frac{1}{2}\left(b_{1}(x)-i \theta b_{2}(x)\right)
$$

is the off-diagonal component. Let

$$
h_{\mathbb{Z}_{2}}=h_{0}+U
$$

Under Assumption 2.2, $U$ is symmetric, relatively bounded with respect to $h_{0}$ with a relative bound strictly smaller than 1 so that $h_{\mathbb{Z}_{2}}$ and $h$ are unitary equivalent,

$$
h_{\mathbb{Z}_{2}} \cong h
$$

as seen below. Define the unitary operator $\mathrm{F}: L^{2}\left(\mathbb{R}^{3} \times \mathbb{Z}_{2}\right) \rightarrow \mathbb{C}^{2} \otimes L^{2}\left(\mathbb{R}^{3}\right)$ by

$$
\mathrm{F}: f \mapsto\left[\begin{array}{l}
f(\cdot,+1) \\
f(\cdot,-1)
\end{array}\right]
$$

Also, define $\tau_{\mu}=F^{-1} \sigma_{\mu} F$. We see that $\tau_{1}: f(x, \theta) \mapsto f(x,-\theta), \tau_{2}: f(x, \theta) \mapsto$ $-i \theta f(x,-\theta)$ and $\tau_{3}: f(x, \theta) \mapsto \theta f(x, \theta)$.

Definition 2.4. Let Assumptions 2.1 and 2.2 hold. Then $H_{\mathbb{Z}_{2}}$ is defined by

$$
H_{\mathbb{Z}_{2}}=\sqrt{2 h_{\mathbb{Z}_{2}}+m^{2}}-m .
$$

In what follows instead of $H$ we study $H_{\mathbb{Z}_{2}}$, and write $H$ (resp. $h$ ) instead of $H_{\mathbb{Z}_{2}}$ (resp. $h_{\mathbb{Z}_{2}}$ ).

\section{$\S 2.3$. Three independent stochastic processes}

In order to construct a path integral representation we will need three independent stochastic processes $\left(B_{t}\right)_{t \geq 0},\left(N_{t}\right)_{t \geq 0}$ and $\left(T_{t}\right)_{t \geq 0}$ which we introduce next. We denote the expectation with respect to path measure $W$ starting at $x$ by $\mathbb{E}_{W}^{x}$.

Let $\left(B_{t}\right)_{t \geq 0}$ be a three-dimensional Brownian motion on a probability space $\left(\Omega_{P}, \mathscr{F}_{P}, P^{x}\right)$ with initial point $P^{x}\left(B_{0}=x\right)=1$.

Secondly, let $\left(N_{t}\right)_{t \geq 0}$ be a Poisson process on a probability space $\left(\Omega_{N}, \mathscr{F}_{N}, \mu\right)$ with unit intensity, i.e.,

$$
\mu\left(N_{t}=n\right)=\frac{t^{n}}{n !} e^{-t}, \quad n \in \mathbb{N} \cup\{0\}
$$

Let $\mu^{\alpha}$ be the image measure of the process $\left(N_{t}+\alpha\right)_{t \geq 0}$ for $\alpha \in \mathbb{Z}_{2}$ and thus $\mathbb{E}_{\mu}^{\alpha}[f(N)]=.\mathbb{E}_{\mu}^{0}[f(N .+\alpha)]$. We define integrals with respect to this process in terms of the sum of evaluations at jumping times, i.e., for $g$ we define

$$
\int_{(a, b]} g\left(s, N_{s}\right) d N_{s}=\sum_{\substack{r \in(a, b] \\ N_{r+} \neq N_{r-}}} g\left(r, N_{r}\right)
$$


and we also write $\int_{a}^{b+} \cdots d N_{s}$ for $\int_{(a, b]} \cdots d N_{s}$. Associated with the Poisson process we also define a $\mathbb{Z}_{2}$-valued stochastic process $\left(\theta_{t}\right)_{t \geq 0}$ on $\left(\Omega_{N}, \mathscr{F}_{N}, \mu^{0}\right)$ by

$$
\theta_{t}=(-1)^{N_{t}}
$$

Finally, let $\left(T_{t}\right)_{t \geq 0}$ denote the subordinator starting from 0 at $t=0$ on a given probability space $\left(\Omega_{\nu}, \mathscr{F}_{\nu}, \nu\right)$ defined by its Laplace transform

$$
\mathbb{E}_{\nu}^{0}\left[e^{-T_{t} u}\right]=\exp \left(-t\left(\sqrt{2 u+m^{2}}-m\right)\right) .
$$

Note that $\left(T_{t}\right)_{t \geq 0}$ is a one-dimensional Lévy process with right continuous paths with left limits, almost surely non-decreasing. It can be more explicitly described as the first hitting time process

$$
T_{t}=\inf \left\{s>0 \mid B_{s}^{1}+m s=t\right\}
$$

where $\left(B_{t}^{1}\right)_{t \geq 0}$ is a one-dimensional Brownian motion independent of the threedimensional Brownian motion $B_{t}$ above. We also define the measure $\nu^{s}, s \in \mathbb{R}$, to be the image measure on $\left(T_{t}+s\right)_{t \geq 0}$, and use the shorthand

$$
\mathbb{E}_{P}^{x} \mathbb{E}_{\mu}^{\alpha} \mathbb{E}_{\nu}^{s}=\mathbb{E}_{M}^{x, \alpha, s}
$$

The role of these three stochastic processes is as follows. Clearly, the Schrödinger operator $-\frac{1}{2} \Delta+V$ generates an Itô process which can be described using the Brownian motion $\left(B_{t}\right)_{t \geq 0}$ under $V$. The Poisson process $\left(N_{t}\right)_{t \geq 0}$ results from the Schrödinger operator with spin. Finally, the subordinator $\left(T_{t}\right)_{t \geq 0}$ appears due to the relativistic Schrödinger operator which generates a Lévy process. A particular combination of these three independent stochastic processes then yields the path integral representation of $e^{-t(H+V)}$ which we will discuss below.

\section{§2.4. Generator of Markov process}

Consider the $\mathbb{R}^{3} \times \mathbb{Z}_{2}$-valued joint Brownian and jump process

$$
\Omega_{P} \times \Omega_{N} \ni\left(\omega, \omega_{1}\right) \mapsto X_{t}\left(\omega, \omega_{1}\right)=\left(B_{t}(\omega), \theta_{t}\left(\omega_{1}\right)\right) \in \mathbb{R}^{3} \times \mathbb{Z}_{2}
$$

with initial value $X_{0}$. The generator of this Markov process is [HIL09]

$$
G_{0}=-\frac{1}{2} \Delta+\sigma_{\mathrm{F}}+1
$$

where 1 is the $2 \times 2$ identity matrix and $\sigma_{\mathrm{F}}$ the fermionic harmonic oscillator defined in terms of the Pauli matrices by

$$
\sigma_{\mathrm{F}}=\frac{1}{2}\left(\sigma_{3}+i \sigma_{2}\right)\left(\sigma_{3}-i \sigma_{2}\right)-1=-\sigma_{1}
$$

Note that inf $\operatorname{Spec}\left(G_{0}\right)=0$. 
In the relativistic case, the subordinator explained above appears in addition to this. We define the subordinate process $\left(q_{t}\right)_{t \geq 0}$ in terms of the $\mathbb{R}^{3} \times \mathbb{Z}_{2}$-valued stochastic process

$$
\begin{aligned}
\Omega_{P} \times \Omega_{N} \times \Omega_{\nu} \ni & \left(\omega, \omega_{1}, \omega_{2}\right) \\
& \mapsto q_{t}\left(\omega, \omega_{1}, \omega_{2}\right)=\left(B_{T_{t}\left(\omega_{2}\right)}(\omega), \theta_{T_{t}\left(\omega_{2}\right)}\left(\omega_{1}\right)\right) \in \mathbb{R}^{3} \times \mathbb{Z}_{2} .
\end{aligned}
$$

In a similar manner to $\left(X_{t}\right)_{t \geq 0}$, we can identify the generator of $\left(q_{t}\right)_{t \geq 0}$.

Proposition 2.5. The generator of the Markov process $\left(q_{t}\right)_{t \geq 0}$ is

$$
G=\sqrt{-\Delta+2 \sigma_{\mathrm{F}}+2+m^{2}}-m
$$

and its characteristic function is given by

$$
\begin{aligned}
\mathbb{E}_{M}^{0,0,0}\left[e^{i Z q_{t}}\right] & =\mathbb{E}_{M}^{0,0,0}\left[e^{i \xi B_{T_{t}}} e^{i z \theta_{T_{t}}}\right] \\
& =e^{-t\left(\sqrt{|\xi|^{2}+m^{2}}-m\right)} \cos z+i e^{-t\left(\sqrt{|\xi|^{2}+4+m^{2}}-m\right)} \sin z
\end{aligned}
$$

for $Z=(\xi, z) \in \mathbb{R}^{3} \times \mathbb{R}$.

Proof. This is obtained through the equalities

$$
\begin{aligned}
\sum_{\alpha=0,1} \int_{\mathbb{R}^{3}} d x \mathbb{E}_{M}^{x, \alpha, 0}\left[\overline{f\left(q_{0}\right)} g\left(q_{t}\right)\right] & =\mathbb{E}_{\nu}^{0}\left[\sum_{\alpha=0,1} \int_{\mathbb{R}^{3}} d x \mathbb{E}_{P \times \mu}^{x, \alpha}\left[\overline{f\left(q_{0}\right)} g\left(q_{t}\right)\right]\right] \\
& =\mathbb{E}_{\nu}^{0}\left[\left(f, e^{-T_{t}\left(-\frac{1}{2} \Delta+\sigma_{F}+1\right)} g\right)\right]=\left(f, e^{-t G} g\right) .
\end{aligned}
$$

Hence (24) is the generator of $\left(q_{t}\right)_{t \geq 0}$, while (25) is straightforward.

\section{§3. Feynman-Kac type representations}

\section{$\S 3.1$. Bounded magnetic field}

In this subsection we briefly discuss some results established in [HIL09] obtained for a general version of the relativistic Schrödinger operator with spin and bounded magnetic field. Write

$$
W(x)=\frac{1}{2} \sqrt{b_{1}(x)^{2}+b_{2}(x)^{2}},
$$

and notice that $\left|U_{\text {od }}(x, \theta)\right|=W(x)$.

Proposition 3.1 (Feynman-Kac formula: bounded magnetic field). Suppose that Assumption 2.1 holds and $b_{\mu} \in L^{\infty}$ for $\mu=1,2,3$. Let $V$ be relatively bounded 
with respect to $\sqrt{-\Delta+m^{2}}$ with a relative bound strictly smaller than 1 . Assume, furthermore, that

$$
\mathbb{E}_{P \times \nu}^{x, 0}\left[\int_{0}^{T_{t}}\left|\log W\left(B_{s}\right)\right| d s\right]<\infty, \quad \text { a.e. } x \in \mathbb{R}^{3} .
$$

Then $H+V$ is self-adjoint on $D(H)$ and

$$
\left(f, e^{-t(H+V)} g\right)=\sum_{\alpha=0,1} \int_{\mathbb{R}^{3}} d x \mathbb{E}_{M}^{x, \alpha, 0}\left[e^{T_{t}} \overline{f\left(q_{0}\right)} g\left(q_{t}\right) e^{\mathscr{S}}\right],
$$

where the exponent $\mathscr{S}=\mathscr{S}_{V}+\mathscr{S}_{\mathrm{A}}+\mathscr{S}_{\mathrm{S}}$ is given by

$$
\begin{aligned}
& \mathscr{S}_{V}=-\int_{0}^{t} V\left(B_{T_{s}}\right) d s, \\
& \mathscr{S}_{\mathrm{A}}=-i \int_{0}^{T_{t}} a\left(B_{s}\right) \circ d B_{s}, \\
& \mathscr{S}_{\mathrm{S}}=-\int_{0}^{T_{t}} U_{\mathrm{d}}\left(B_{s}, \theta_{s}\right) d s+\int_{0}^{T_{t}+} \log \left(-U_{\text {od }}\left(B_{s},-\theta_{s-}\right)\right) d N_{s} .
\end{aligned}
$$

Proof. Since $\|V f\| \leq \kappa\left\|\sqrt{-\Delta+m^{2}} f\right\|+\kappa^{\prime}\|f\|$ with constants $\kappa<1$ and $\kappa^{\prime}$, and $b_{\mu}$ is bounded, we have $\|V f\| \leq \kappa\|H f\|+C\|f\|$ with a constant $C$. Hence self-adjointness follows by the Kato-Rellich theorem. (28) follows from [HIL09, Theorem 5.9].

We note that $\mathscr{S}_{\mathrm{A}}$ and $\mathscr{S}_{\mathrm{S}}$ in Proposition 3.1 stand for the stochastic integrals $-i \int_{0}^{r} a\left(B_{s}\right) \circ d B_{s}$ and $-\int_{0}^{r} U_{\mathrm{d}}\left(B_{s}, \theta_{s}\right) d s+\int_{0}^{r+} \log \left(-U_{\mathrm{od}}\left(B_{s},-\theta_{s-}\right)\right) d N_{s}$ evaluated at $r=T_{t}$, respectively.

A Feynman-Kac formula without spin is an immediate corollary. This was first established in [CMS90] without a vector potential; we give a version including a vector potential. Let

$$
H_{\text {spinless }}=\sqrt{2 h_{0}+m^{2}}-m \text {. }
$$

Corollary 3.2. Let Assumption 2.1 hold, and assume that $V=V_{+}-V_{-}$is such that $V_{+} \in L_{\mathrm{loc}}^{1}\left(\mathbb{R}^{3}\right)$ and $V_{-}$is relatively form bounded with respect to $\sqrt{-\Delta+m^{2}}$ with a relative bound strictly less than 1 . Then

$$
\left(f, e^{-t\left(H_{\text {spinless }} \dot{+} V_{+} \dot{-} V_{-}\right)} g\right)=\int_{\mathbb{R}^{3}} d x \mathbb{E}_{P \times \nu}^{x, 0}\left[\overline{f\left(B_{0}\right)} g\left(B_{T_{t}}\right) e^{\mathscr{S}_{V}+\mathscr{S}_{\mathrm{A}}}\right] .
$$

In particular, when $a=0$,

$$
\left(f, e^{-t\left(\sqrt{-\Delta+m^{2}}-m \dot{+} V_{+} \dot{-} V_{-}\right)} g\right)=\int_{\mathbb{R}^{3}} d x \mathbb{E}_{P \times \nu}^{x, 0}\left[\overline{f\left(X_{0}\right)} g\left(X_{t}\right) e^{-\int_{0}^{t} V\left(X_{s}\right) d s}\right],
$$

where $\left(X_{t}\right)_{t \geq 0}$ is the stochastic process defined in (21). 
By Corollary 3.2 we have the following energy comparison inequality with

$$
H_{0}=\sqrt{-\Delta+m^{2}}-m \text {. }
$$

Corollary 3.3. Under the assumptions of Corollary 3.2 we have

(1) $\left|\left(f, e^{-t\left(H_{\text {spinless }} \dot{+} V_{+} \dot{-} V_{-}\right)} g\right)\right| \leq\left(|f|, e^{-t\left(H_{0} \dot{+} V_{+} \dot{-} V_{-}\right)}|g|\right)$,

(2) $\inf \operatorname{Spec}\left(H_{0} \dot{+} V_{+} \dot{-} V_{-}\right) \leq \inf \operatorname{Spec}\left(H_{\text {spinless }} \dot{+} V_{+} \dot{-} V_{-}\right)$.

\section{§3.2. Unbounded magnetic field}

We extend the Feynman-Kac formula above (Proposition 3.1) to the case of magnetic fields $b$ that are possibly unbounded and satisfy Assumption 2.2. This extension is not straightforward, and we need several lemmas.

Define the truncated magnetic field $b^{(N)}$ by

$$
b_{\mu}^{(N)}(x)= \begin{cases}b_{\mu}(x) & \text { if }\left|b_{\mu}(x)\right| \leq N, \\ N & \text { if } b_{\mu}(x)>N, \\ -N & \text { if } b_{\mu}(x)<-N .\end{cases}
$$

Then the Feynman-Kac formula for the Hamiltonian with the truncated magnetic field is readily given by Proposition 3.1 in which $b$ is replaced by $b^{(N)}$. Let $H_{N}$ be defined by $H$ with $b$ replaced by $b^{(N)}$.

Lemma 3.4. Under Assumptions 2.1 and 2.2 the semigroup $e^{-t H_{N}}$ is strongly convergent to $e^{-t H}$ as $N \rightarrow \infty$.

Proof. Let $h_{N}$ be $h$ with $b$ replaced by $b^{(N)}$. We see that $h_{N} \rightarrow h$ as $N \rightarrow \infty$ on the common domain $D\left(h_{n}\right)=D(h)$. Then $e^{-t h_{N}} \rightarrow e^{-t h}$ strongly as $N \rightarrow \infty$. Thus it is immediate to see that

$$
\left(f, e^{-t H_{N}} g\right)=\mathbb{E}_{\nu}^{0}\left[\left(f, e^{-T_{t} h_{N}} g\right)\right] \rightarrow \mathbb{E}_{\nu}^{0}\left[\left(f, e^{-T_{t} h} g\right)\right]=\left(f, e^{-t H} g\right),
$$

which implies strong convergence.

Lemma 3.5. Let $f, g \in L^{2}\left(\mathbb{R}^{3} \times \mathbb{Z}_{2}\right)$, and set

$$
\rho=f\left(q_{0}\right) g\left(q_{t}\right) e^{\int_{0}^{T_{t}} \frac{1}{2}\left|b_{3}\left(B_{s}\right)\right| d s} e^{\int_{0}^{T_{t}+} \log W\left(B_{s}\right) d N_{s}} e^{T_{t}} .
$$

Then Assumption 2.2 yields $\sum_{\alpha=0,1} \int_{\mathbb{R}^{3}} d x \mathbb{E}_{M}^{x, \alpha, 0}[|\rho|]<\infty$.

Proof. Define the spin operators $|U|$ and $|U|_{N}$ by

$$
\begin{aligned}
& |U|: f(x, \theta) \mapsto-\frac{1}{2}\left|b_{3}(x)\right| f(x, \theta)-W(x) f(x,-\theta) \\
& |U|_{N}: f(x, \theta) \mapsto-\frac{1}{2}\left|b_{3}^{(N)}(x)\right| f(x, \theta)-W^{(N)}(x) f(x,-\theta),
\end{aligned}
$$


where $W^{(N)}$ is $W$ with $b$ replaced by $b^{(N)}$, and define

$$
\widehat{H}=\sqrt{-\Delta+2|U|+m^{2}}-m .
$$

Also, we define $\widehat{H}_{N}$ to be $\widehat{H}$ with $|U|$ replaced by $|U|_{N}$. Let $f, g \in L^{2}\left(\mathbb{R}^{3}\right)$ be non-negative. For $\widehat{H}_{N}$ we have the Feynman-Kac formula

$$
\left(f, e^{-t \widehat{H}_{N}} g\right)=\sum_{\alpha=0,1} \int_{\mathbb{R}^{3}} d x \mathbb{E}_{M}^{x, \alpha, 0}\left[e^{T_{t}} \overline{f\left(q_{0}\right)} g\left(q_{t}\right) e^{\widehat{\mathscr{P}}_{\mathrm{S}}^{N}}\right],
$$

where

$$
\widehat{\mathscr{S}}_{\mathrm{S}}^{N}=\int_{0}^{T_{t}} \frac{1}{2}\left|b_{3}^{(N)}\left(B_{s}\right)\right| d s+\int_{0}^{T_{t}+} \log W^{(N)}\left(B_{s}\right) d N_{s} .
$$

By the monotone convergence theorem for forms we see that $e^{-t\left(-\Delta+2|U|_{N}\right)} \rightarrow$ $e^{-t(-\Delta+2|U|)}$ strongly as $N \rightarrow \infty$, and then the strong convergence $e^{-t \widehat{H}_{N}} \rightarrow e^{-t \widehat{H}}$ as $N \rightarrow \infty$ is shown in the same way as (36). Then the monotone convergence theorem for integrals implies that $\rho$ is integrable and the Feynman-Kac formula (40) with $b^{(N)}$ replaced by $b$ also holds.

Now we can state the first main theorem.

Theorem 3.6 (Feynman-Kac formula: unbounded magnetic field). Let Assumptions 2.1 and 2.2 as well as condition (27) hold, and suppose that $V$ is relatively bounded with respect to $\sqrt{-\Delta+m^{2}}$ with a relative bound strictly less than 1 . Then $H+V$ is self-adjoint on $D(H)$ and

$$
\left(f, e^{-t(H+V)} g\right)=\sum_{\alpha=0,1} \int_{\mathbb{R}^{3}} d x \mathbb{E}_{M}^{x, \alpha, 0}\left[e^{T_{t}} \overline{f\left(q_{0}\right)} g\left(q_{t}\right) e^{\mathscr{S}}\right] .
$$

Proof. We divide the proof into five steps.

Step 1. Suppose that $V=0$. Then the statement holds.

Proof. Recall that $H_{N}$ is defined like $H$ with $b$ replaced by $b^{(N)}$. Then the Feynman-Kac formula holds with $\mathscr{S}_{\mathrm{S}}$ replaced by $\mathscr{S}_{\mathrm{S}}^{N}$, where $\mathscr{S}_{\mathrm{S}}^{N}$ is defined like $\mathscr{S}_{\mathrm{S}}$ with $b$ replaced by $b^{(N)}$ :

$$
\left(f, e^{-t H_{N}} g\right)=\sum_{\alpha=0,1} \int_{\mathbb{R}^{3}} d x \mathbb{E}_{M}^{x, \alpha, 0}\left[e^{T_{t}} \overline{f\left(q_{0}\right)} g\left(q_{t}\right) e^{\mathscr{S}_{\mathrm{S}}^{N}+\mathscr{S}_{\mathrm{A}}}\right] .
$$

The left hand side above converges to $\left(f, e^{-t H} g\right)$ as $N \rightarrow \infty$ by Lemma 3.4. On the other hand, we have

$$
e^{T_{t}}\left|f\left(q_{0}\right) g\left(q_{t}\right)\right|\left|e^{\mathscr{S}_{\mathrm{S}}^{N}+\mathscr{S}_{\mathrm{A}}}\right| \leq e^{T_{t}}\left|f\left(q_{0}\right) g\left(q_{t}\right)\right| e^{\int_{0}^{T_{t}} \frac{1}{2}\left|b_{3}\left(B_{s}\right)\right| d s} e^{\int_{0}^{T_{t}+} \log W\left(B_{s}\right) d N_{s}}
$$


so that the right hand side of (43) is integrable by Lemma 3.5, and therefore the Lebesgue dominated convergence theorem yields

$$
\begin{aligned}
\lim _{N \rightarrow \infty} \sum_{\alpha=0,1} \int_{\mathbb{R}^{3}} d x \mathbb{E}_{M}^{x, \alpha, 0}\left[e^{T_{t}}\right. & \left.f\left(q_{0}\right) g\left(q_{t}\right) e^{\mathscr{S}_{\mathrm{S}}^{N}+\mathscr{S}_{\mathrm{A}}}\right] \\
& =\sum_{\alpha=0,1} \int_{\mathbb{R}^{3}} d x \mathbb{E}_{M}^{x, \alpha, 0}\left[e^{T_{t}} f\left(q_{0}\right) g\left(q_{t}\right) e^{\mathscr{S}_{\mathrm{S}}+\mathscr{S}_{\mathrm{A}}}\right] .
\end{aligned}
$$

Hence the statement of the theorem follows for $V=0$.

Step 2. $V$ is relatively bounded with respect to $H$ with a relative bound strictly smaller than 1. In particular, $H+V$ is self-adjoint on $D(H)$.

Proof. Let $b_{0}=\left(\sqrt{b_{1}^{2}+b_{2}^{2}}, 0, b_{3}\right)$ and $H_{b_{0}}$ be defined by $H$ with $a=0$ and $b$ replaced by $b_{0}$, i.e., $H_{b_{0}}=\sqrt{-\Delta+\sigma \cdot b_{0}+m^{2}}-m$. Set $\sigma \cdot b_{0}=U_{b_{0}}$. Then we have

$$
\left\|\sqrt{-\Delta+m^{2}} f\right\|^{2}=\left\|\left(H_{b_{0}}+m\right) f\right\|^{2}+\left(f,-U_{b_{0}} f\right) .
$$

Since $\left|\left(f, U_{b_{0}} f\right)\right| \leq \kappa^{\prime}\|f\|^{2}$ with a constant $\kappa^{\prime}$, and $\|V f\| \leq \kappa\left\|\sqrt{-\Delta+m^{2}} f\right\|+$ $\kappa^{\prime \prime}\|f\|$ with constants $\kappa<1$ and $\kappa^{\prime \prime}$, we have $\|V f\| \leq A\left\|H_{b_{0}} f\right\|+C\|f\|$ with some $C$ and $A<1$. The Feynman-Kac formula established in Step 1 implies the diamagnetic inequality

$$
\left|\left(f, e^{-t H} g\right)\right| \leq\left(|f|, e^{-t H_{b_{0}}}|g|\right) .
$$

From (44) we have $\left\|H_{b_{0}} f\right\| \leq\|H f\|+c\|f\|$, and thus

$$
\|V f\| \leq A\|H f\|+C^{\prime}\|f\|
$$

with a constant $C^{\prime}$. Hence self-adjointness follows by the Kato-Rellich theorem.

Step 3. Suppose $V \in L^{\infty}\left(\mathbb{R}^{3}\right) \cap C\left(\mathbb{R}^{3}\right)$. Then the statement holds.

Proof. By the Trotter product formula and the Markov property of $\left(q_{t}\right)_{t \geq 0}$ we have

$$
\begin{aligned}
\left(f, e^{-t(H+V)} g\right) & =\lim _{n \rightarrow \infty}\left(f,\left(e^{-(t / n) H} e^{-(t / n) V}\right)^{n} g\right) \\
& =\sum_{\alpha=0,1} \int_{\mathbb{R}^{3}} d x \mathbb{E}_{M}^{x, \alpha, 0}\left[e^{T_{t}} f\left(q_{0}\right) g\left(q_{t}\right) e^{-\sum_{j=1}^{n}(t / n) V\left(B_{T_{t j} / n}\right)} e^{\mathscr{S}_{\mathrm{S}}+\mathscr{S}_{\mathrm{A}}}\right] .
\end{aligned}
$$

Note that $s \mapsto V\left(B_{T_{s}}\right)$ is continuous in $s \in[0, t]$ except possibly at finitely many points. Thus

$$
-\sum_{j=1}^{n}(t / n) V\left(B_{T_{t j / n}\left(\omega_{2}\right)}(\omega)\right) \stackrel{n \rightarrow \infty}{\longrightarrow}-\int_{0}^{t} V\left(B_{T_{s}\left(\omega_{2}\right)}(\omega)\right) d s
$$


for almost every $\left(\omega, \omega_{2}\right) \in \Omega_{P} \times \Omega_{\nu}$ as a Riemann integral. Then the conclusion of the theorem follows for $V \in L^{\infty}\left(\mathbb{R}^{3}\right) \cap C\left(\mathbb{R}^{3}\right)$.

Step 4. Suppose $V \in L^{\infty}\left(\mathbb{R}^{3}\right)$. Then the statement holds.

Proof. Let $V_{n}=\phi(\cdot / n)\left(V * j_{n}\right)$, where $j_{n}(x)=n^{3} \phi(x n)$ with $\phi \in C_{0}^{\infty}$ such that $0 \leq \phi \leq 1, \int_{\mathbb{R}^{3}} \phi(x) d x=1$ and $\phi(0)=1$. Then $V_{n}(x) \rightarrow V(x)$ for $x \notin \mathscr{N}$, where $\mathscr{N}$ is a set of Lebesgue measure zero. Notice that

$$
\mathbb{E}_{P \times \nu}^{x, 0}\left[1_{\mathscr{N}}\left(B_{T_{s}}\right)\right]=\int_{\mathbb{R}^{3}} 1_{\mathscr{N}}(x+y) \mathrm{P}_{s}(y) d y=0
$$

for $x \in \mathscr{N}$, where

$$
\mathrm{P}_{s}(x)=2\left(\frac{m}{2 \pi}\right)^{2} \frac{s}{s^{2}+|x|^{2}} K_{2}\left(m \sqrt{|x|^{2}+s^{2}}\right)
$$

is the distribution of the random variable $B_{T_{s}}$ and

$$
K_{2}(x)=\frac{1}{2} \int_{0}^{\infty} \xi e^{-\frac{1}{2}\left(\xi+\xi^{-1}\right) x} d \xi
$$

is the modified Bessel function of the third kind. Hence

$$
0=\int_{0}^{t} \mathbb{E}_{P \times \nu}^{x, 0}\left[1_{\mathscr{N}}\left(B_{T_{s}}\right)\right] d s=\mathbb{E}_{P \times \nu}^{x, 0}\left[\int_{0}^{t} 1_{\mathscr{N}}\left(B_{T_{s}}\right) d s\right] .
$$

Then the Lebesgue measure of $\left\{s \in[0, \infty) \mid B_{T_{s}\left(\omega_{2}\right)}(\omega) \in \mathscr{N}\right\}$ is zero for almost every path $\left(\omega, \omega_{2}\right) \in \Omega_{P} \times \Omega_{\nu}$. Therefore $\int_{0}^{t} V_{n}\left(B_{T_{s}}\right) d s \rightarrow \int_{0}^{t} V\left(B_{T_{s}}\right) d s$ as $n \rightarrow \infty$ for almost every path $\left(\omega, \omega_{2}\right) \in \Omega_{P} \times \Omega_{\nu}$. Moreover,

$$
\begin{aligned}
\sum_{\alpha=0,1} \int_{\mathbb{R}^{3}} d x \mathbb{E}_{M}^{x, \alpha, 0}\left[e^{T_{t}} \overline{f\left(q_{0}\right)} g\left(q_{t}\right) e^{\mathscr{S}_{\mathrm{A}}+\mathscr{S}_{\mathrm{S}}} e^{-\int_{0}^{t} V_{n}\left(B_{s}\right) d s}\right] \\
\stackrel{n \rightarrow \infty}{\longrightarrow} \sum_{\alpha=0,1} \int_{\mathbb{R}^{3}} d x \mathbb{E}_{M}^{x, \alpha, 0}\left[e^{T_{t}} \overline{f\left(q_{0}\right)} g\left(q_{t}\right) e^{\mathscr{S}_{\mathrm{A}}+\mathscr{S}_{\mathrm{S}}} e^{-\int_{0}^{t} V\left(B_{s}\right) d s}\right] .
\end{aligned}
$$

On the other hand, $e^{-t\left(H+V_{n}\right)} \rightarrow e^{-t(H+V)}$ strongly as $n \rightarrow \infty$, since $H+V_{n}$ converges to $H+V$ on the common domain $D(H)$. Then the conclusion follows for $V \in L^{\infty}\left(\mathbb{R}^{3}\right)$.

Step 5. We complete the proof of Theorem 3.6. Let $V=V_{+}-V_{-}$and $V_{m n}=$ $V_{+m}-V_{-n}$, with $V_{+}, V_{-}$denoting the positive and negative parts of $V$, respectively, and $V_{+m}(x)=V_{+}(x)$ if $V_{+}(x) \leq m$, and $V_{+}(x)=m$ if $V_{+}(x) \geq m$, similarly $V_{-n}(x)=V_{-}(x)$ if $V_{-}(x) \leq n$ and $V_{-}(x)=n$ if $V_{-}(x) \geq n$. Then by the monotone convergence theorem for forms, $e^{-t\left(H+V_{m n}\right)}$ strongly converges to $e^{-t\left(H+V_{m \infty}\right)}$ as 
$n \rightarrow \infty$, and $e^{-t\left(H+V_{m} \infty\right)}$ strongly converges to $e^{-t(H+V)}$ as $m \rightarrow \infty$. Hence

$$
\lim _{m \rightarrow \infty} \lim _{n \rightarrow \infty} e^{-t\left(H+V_{m n}\right)}=e^{-t(H+V)} .
$$

On the other hand, by the monotone convergence theorem for integrals, we have

$$
\begin{aligned}
\lim _{m \rightarrow \infty} \lim _{n \rightarrow \infty} \sum_{\alpha=0,1} & \int_{\mathbb{R}^{3}} d x \mathbb{E}_{M}^{x, \alpha, 0}\left[e^{T_{t}} \overline{f\left(q_{0}\right)} g\left(q_{t}\right) e^{\mathscr{S}_{\mathrm{A}}+\mathscr{S}_{\mathrm{S}}} e^{-\int_{0}^{t} V_{m n}\left(B_{s}\right) d s}\right] \\
= & \sum_{\alpha=0,1} \int_{\mathbb{R}^{3}} d x \mathbb{E}_{M}^{x, \alpha, 0}\left[e^{T_{t}} \overline{f\left(q_{0}\right)} g\left(q_{t}\right) e^{\mathscr{S}_{\mathrm{A}}+\mathscr{S}_{\mathrm{S}}} e^{-\int_{0}^{t} V\left(B_{s}\right) d s}\right] .
\end{aligned}
$$

This completes the proof of the theorem.

\section{§3.3. Magnetic field with zeros}

Next we consider the case when the off-diagonal component $U_{\text {od }}(x,-\theta)$ vanishes for some $x \in \mathbb{R}^{3}$. In this case it is not clear whether $\int_{0}^{t+}\left|\log W\left(B_{s}\right)\right| d N_{s}<\infty$ almost surely. An example when this is not the case is obtained by choosing $b \in\left(C_{0}^{\infty}\right)^{3}$.

Let

$$
\delta_{\varepsilon}(z)= \begin{cases}1, & |z|<\varepsilon \\ 0, & |z| \geq \varepsilon\end{cases}
$$

for $z \in \mathbb{C}$ and write

$$
\chi_{\varepsilon}(z)=z+\varepsilon \delta_{\varepsilon}(z), \quad z \in \mathbb{C}
$$

We see that

$$
\left|\chi_{\varepsilon}\left(U_{\text {od }}(x,-\theta)\right)\right|>\varepsilon, \quad(x, \theta) \in \mathbb{R}^{3} \times \mathbb{Z}_{2} .
$$

Define $h_{\varepsilon}$ to be $h$ with the off-diagonal part replaced by $\chi_{\varepsilon}\left(U_{\text {od }}(x,-\theta)\right)$, i.e.,

$$
h_{\varepsilon} f(x, \theta)=\left(h_{0}+U_{\mathrm{d}}(x, \theta)\right) f(x, \theta)+\chi_{\varepsilon}\left(U_{\text {od }}(x,-\theta)\right) f(x,-\theta), \quad(x, \theta) \in \mathbb{R}^{3} \times \mathbb{Z}_{2} .
$$

Also, define $H_{\varepsilon}$ to be $H$ with $U_{\text {od }}$ replaced by $\chi_{\varepsilon}\left(U_{\text {od }}(x,-\theta)\right)$.

We note that for every $\left(x, \omega, \omega_{1}, \omega_{2}\right) \in \mathbb{R}^{3} \times \Omega_{P} \times \Omega_{N} \times \Omega_{\nu}$ there exists a number $n=n\left(\omega_{1}, \omega_{2}\right)$ and random jump times $r_{1}\left(\omega_{1}\right), \ldots, r_{n}\left(\omega_{1}\right)$ of $s \mapsto N_{s}$ for $0 \leq s \leq T_{t}\left(\omega_{2}\right)$ such that

$$
\int_{0}^{T_{t}\left(\omega_{2}\right)+} \log W\left(x+B_{s}(\omega)\right) d N_{s}=\sum_{j=1}^{n\left(\omega_{1}, \omega_{2}\right)} \log W\left(x+B_{r_{j}\left(\omega_{1}\right)}(\omega)\right) .
$$

Consider

(46)

$$
\mathscr{W}=\left\{\left(x, \omega, \omega_{1}, \omega_{2}\right) \in \mathbb{R}^{3} \times \Omega_{P} \times \Omega_{N} \times \Omega_{\nu} \mid \int_{0}^{T_{t}+} \log W\left(x+B_{s}\right) d N_{s}>-\infty\right\} .
$$


By definition, $\left(x, \omega, \omega_{1}, \omega_{2}\right) \in \mathscr{W}^{c}$ if and only if there exists $r$ such that

(1) $0<r \leq t \leq T_{t}\left(\omega_{2}\right)$,

(2) $s \mapsto N_{s}$ is discontinuous at $s=r$,

(3) $b_{1}\left(B_{r}(\omega)\right)=b_{2}\left(B_{r}(\omega)\right)=0$.

Lemma 3.7. For every $\left(x, \omega, \omega_{1}, \omega_{2}\right) \in \mathscr{W}^{c}$ we have

$$
\lim _{\varepsilon \rightarrow 0}\left|e^{\int_{0}^{T_{t}+} \log \left(-\chi_{\varepsilon}\left(U_{\text {od }}\left(B_{s},-\theta_{s-}\right)\right) d N_{s}\right.}\right|=0 .
$$

Proof. We have $\left|e^{\int_{0}^{T_{t}+} \log \left(-\chi_{\varepsilon}\left(U_{\text {od }}\left(B_{s},-\theta_{s-}\right)\right)\right) d N_{s}}\right| \leq e^{\int_{0}^{T_{t}+} \log \left(W\left(B_{s}\right)+\varepsilon\right) d N_{s}}$. Observe that

$$
\int_{0}^{T_{t}+} \log \left(W\left(B_{s}\right)+\varepsilon\right) d N_{s}=\sum_{j=1}^{n} \log \left(W\left(B_{r_{j}}\right)+\varepsilon\right), \quad r_{1}, \ldots, r_{n} \in\left(0, T_{t}\right] .
$$

Since $\left(x, \omega, \omega_{1}, \omega_{2}\right) \in \mathscr{W}^{c}$, there exists an $r_{i}$ such that $b_{1}\left(B_{r_{i}}(\omega)\right)=b_{2}\left(B_{r_{i}}(\omega)\right)=0$. Then

$$
\int_{0}^{T_{t}+} \log \left(W\left(B_{s}\right)+\varepsilon\right) d N_{s}=\sum_{j \neq i}^{n} \log \left(W\left(B_{r_{j}}\right)+\varepsilon\right)+\log \varepsilon
$$

and $e^{\int_{0}^{T_{t}+} \log \left(W\left(B_{s}\right)+\varepsilon\right) d N_{s}} \leq e^{\sum_{j \neq i}^{n} \log \left(W\left(B_{r_{j}}\right)+\varepsilon\right)} e^{\log \varepsilon}$. Thus

$$
\lim _{\varepsilon \rightarrow 0}\left|e^{\int_{0}^{T_{t}+} \log \left(W\left(B_{s}\right)+\varepsilon\right) d N_{s}}\right|=0,
$$

and the lemma follows.

Theorem 3.8 (Feynman-Kac formula: magnetic field with zeros). Let Assumptions 2.1 and 2.2 hold, and suppose that $V$ is relatively bounded with respect to $\sqrt{-\Delta+m^{2}}$ with a relative bound strictly less than 1 . Let $\mathscr{W}$ be given by (46). Then

$$
\left(f, e^{-t(H+V)} g\right)=\sum_{\alpha=0,1} \int_{\mathbb{R}^{3}} d x \mathbb{E}_{M}^{x, \alpha, 0}\left[e^{T_{t}} \overline{f\left(q_{0}\right)} g\left(q_{t}\right) e^{\mathscr{S}} 1_{\mathscr{W}}\right] .
$$

Proof. Put $V=0$ and fix $\varepsilon>0$. We can show that the functional integral representation of $H_{\varepsilon}$ is given by (42) with $\mathscr{S}$ replaced by $\mathscr{S}_{\mathrm{A}}+\mathscr{S}_{\mathrm{S}}(\varepsilon)$ with

$$
\mathscr{S}_{\mathrm{S}}(\varepsilon)=-\int_{0}^{T_{t}} U_{\mathrm{d}}\left(B_{s}, \theta_{s}\right) d s+\int_{0}^{T_{t}+} \log \left(-\chi_{\varepsilon}\left(U_{\mathrm{od}}\left(B_{s},-\theta_{s-}\right)\right)\right) d N_{s} .
$$

That is,

$$
\left(f, e^{-t H_{\varepsilon}} g\right)=\sum_{\alpha=0,1} \int_{\mathbb{R}^{3}} d x \mathbb{E}_{M}^{x, \alpha, 0}\left[e^{T_{t}} \overline{f\left(q_{0}\right)} g\left(q_{t}\right) e^{\mathscr{S}_{\mathrm{A}}+\mathscr{S}_{\mathrm{S}}(\varepsilon)}\right]
$$


Take the limit $\varepsilon \downarrow 0$ on both sides above. This gives

$$
\lim _{\varepsilon \downarrow 0} \exp \left(-t H_{\varepsilon}\right)=\exp (-t H)
$$

in strong sense, obtained in the same way as Lemma 3.4. On the other hand, by the Lebesgue dominated convergence theorem it follows that

$$
\begin{aligned}
& \lim _{\varepsilon \downarrow 0} \int_{\mathbb{R}^{3}} d x \mathbb{E}_{M}^{x, \alpha, 0}\left[e^{T_{t}} \overline{f\left(q_{0}\right)} g\left(q_{t}\right) e^{\mathscr{S}_{\mathrm{A}}+\mathscr{S}_{\mathrm{S}}(\varepsilon)}\right] \\
&=\int_{\mathbb{R}^{3}} d x \mathbb{E}_{M}^{x, \alpha, 0}\left[\lim _{\varepsilon \downarrow 0} e^{T_{t}} \overline{f\left(q_{0}\right)} g\left(q_{t}\right) e^{\mathscr{S}_{\mathrm{A}}+\mathscr{S}_{\mathrm{S}}(\varepsilon)}\right] .
\end{aligned}
$$

By Lemma 3.7 we find that $\lim _{\varepsilon \rightarrow 0} \mathscr{S}_{\mathrm{S}}(\varepsilon)=0$ on $\mathscr{W}$ and hence

$$
\lim _{\varepsilon \downarrow 0} e^{\mathscr{S}_{\mathrm{A}}+\mathscr{S}_{\mathrm{S}}(\varepsilon)}=\lim _{\varepsilon \downarrow 0} e^{\mathscr{S}_{\mathrm{A}}+\mathscr{S}_{\mathrm{S}}(\varepsilon)} 1_{\mathscr{W}}+\lim _{\varepsilon \downarrow 0} e^{\mathscr{S}_{\mathrm{A}}+\mathscr{S}_{\mathrm{S}}(\varepsilon)} 1_{\mathscr{W} c}=e^{\mathscr{S}_{\mathrm{A}}+\mathscr{S}_{\mathrm{S}}} 1_{\mathscr{W}} .
$$

Next suppose that $V \in L^{\infty}\left(\mathbb{R}^{3}\right) \cap C\left(\mathbb{R}^{3}\right)$. In this case we can show the theorem in the same way as in Step 3 in the proof of Theorem 3.6. Furthermore, the theorem holds for the required $V$ in the same way as in Steps 4 and 5 above.

A diamagnetic inequality follows immediately from Theorem 3.8. Recall that $H_{b_{0}}$ is defined to be $H$ with $b$ replaced by $b_{0}=\left(\sqrt{b_{1}^{2}+b_{2}^{2}}, 0, b_{3}\right)$ and $a$ by zero, respectively.

Corollary 3.9 (Energy comparison inequality). Under the assumptions of Theorem 3.8 we have

$$
\left|\left(f, e^{-t(H+V)} g\right)\right| \leq\left(|f|, e^{-t\left(H_{b_{0}}+V\right)}|g|\right) .
$$

In particular, $\inf \operatorname{Spec}\left(H_{b_{0}}+V\right) \leq \inf \operatorname{Spec}(H+V)$.

\section{§4. Fall-off of bound states}

\section{§4.1. Martingale properties: non-relativistic case}

In this section we shall prove the decay properties of bound states of relativistic Schrödinger operators with spin by means of the Feynman-Kac formula derived in the previous section. For simplicity we assume throughout that

$$
\mathbb{E}_{P}^{x}\left[\int_{0}^{t}\left|\log W\left(B_{s}\right)\right| d s\right]<\infty, \quad \text { a.e. } x \in \mathbb{R}^{3},
$$

and

$$
\mathbb{E}_{P \times \nu}^{x, 0}\left[\int_{0}^{T_{t}}\left|\log W\left(B_{s}\right)\right| d s\right]<\infty, \quad \text { a.e. } x \in \mathbb{R}^{3},
$$

i.e., the measure of $\mathscr{W}^{c}$ in (46) is zero. 
We first consider the non-relativistic case. Let $H_{\mathrm{NR}}$ be the Hamiltonian defined by

$$
H_{\mathrm{NR}}=h+V,
$$

where $h$ is given by (7). Let $\mathscr{S}_{\mathrm{NR}}$ be defined to be the exponent $\mathscr{S}$ with the subordinator $T_{t}$ replaced by the non-random time $t$. If Assumptions 2.1 and 2.2 hold and $V$ is relatively bounded with respect to $-\Delta$ with a relative bound strictly smaller than 1, then $h+V$ is self-adjoint on $D(-\Delta)$. Then the Feynman-Kac formula of $\left(f, e^{-t(h+V)} g\right)$ is

$$
\left(f, e^{-t(h+V)} g\right)=\sum_{\alpha=0,1} \int_{\mathbb{R}^{3}} d x \mathbb{E}_{P \times \mu}^{x, \alpha}\left[e^{t} \overline{f\left(B_{0}, \theta_{0}\right)} g\left(B_{t}, \theta_{t}\right) e^{\mathscr{S}_{\mathrm{NR}}}\right],
$$

where the exponent $\mathscr{S}_{\mathrm{NR}}=\mathscr{S}_{\mathrm{NR} V}+\mathscr{S}_{\mathrm{NRA}}+\mathscr{S}_{\mathrm{NRS}}$ is given by

$$
\begin{aligned}
& \mathscr{S}_{\mathrm{NR} V}=-\int_{0}^{t} V\left(B_{s}\right) d s, \\
& \mathscr{S}_{\mathrm{NRA}}=-i \int_{0}^{t} a\left(B_{s}\right) \circ d B_{s}, \\
& \mathscr{S}_{\mathrm{NRS}}=-\int_{0}^{t} U_{\mathrm{d}}\left(B_{s}, \theta_{s}\right) d s+\int_{0}^{t+} \log \left(-U_{\mathrm{od}}\left(B_{s},-\theta_{s-}\right)\right) d N_{s} .
\end{aligned}
$$

Let $\varphi_{\mathrm{g}}$ be a bound state such that $H_{\mathrm{NR}} \varphi_{\mathrm{g}}=E \varphi_{\mathrm{g}}$ with $E \in \mathbb{R}$. We consider the spatial decay of $\left|\varphi_{\mathrm{g}}\left(x,(-1)^{\alpha}\right)\right|$, i.e., its behavior for large $|x|$.

Let $\mathscr{S}_{\mathrm{NR}}(x, \alpha)=\mathscr{S}_{\mathrm{NR} V}(x)+\mathscr{S}_{\mathrm{NRA}}(x)+\mathscr{S}_{\mathrm{NRS}}(x, \alpha)$ be $\mathscr{S}_{\mathrm{NR}}$ with $B_{s}$ and $N_{s}$ replaced by $B_{s}+x$ and $N_{s}+\alpha$, respectively:

$$
\begin{aligned}
\mathscr{S}_{\mathrm{NR} V}(x)= & -\int_{0}^{t} V\left(B_{s}+x\right) d s, \\
\mathscr{S}_{\mathrm{NRA}}(x)= & -i \int_{0}^{t} a\left(B_{s}+x\right) \circ d B_{s}, \\
\mathscr{S}_{\mathrm{NRS}}(x, \alpha)= & -\int_{0}^{t} U_{\mathrm{d}}\left(B_{s}+x,(-1)^{\alpha} \theta_{s}\right) d s \\
& +\int_{0}^{t+} \log \left(-U_{\mathrm{od}}\left(B_{s}+x,-(-1)^{\alpha} \theta_{s-}\right)\right) d N_{s} .
\end{aligned}
$$

Define the stochastic process $\left(M_{t}(x, \alpha)\right)_{t \geq 0}$ by

$$
M_{t}(x, \alpha)=e^{t(E+1)} e^{\mathscr{S}_{\mathrm{NR}}(x, \alpha)} \varphi_{\mathrm{g}}\left(B_{t}+x,(-1)^{\alpha} \theta_{t}\right), \quad t \geq 0,
$$

and the filtration

$$
\mathscr{M}_{t}=\sigma\left(\left(B_{r}, \theta_{r}\right), 0 \leq r \leq t\right), \quad t \geq 0 .
$$


Note that $e^{-t\left(H_{\mathrm{NR}}-E\right)} \varphi_{\mathrm{g}}=\varphi_{\mathrm{g}}$ and then

$$
\mathbb{E}_{P \times \mu}^{x, \alpha}\left[M_{t}(0,0)\right]=\mathbb{E}_{P \times \mu}^{0,0}\left[M_{t}(x, \alpha)\right]=\varphi_{\mathrm{g}}\left(x,(-1)^{\alpha}\right)
$$

by $(55)$.

Lemma 4.1. The stochastic process $\left(M_{t}(x, \alpha)\right)_{t \geq 0}$ is a martingale with respect to $\left(\mathscr{M}_{t}\right)_{t \geq 0}$, i.e., $\mathbb{E}_{P \times \mu}^{0,0}\left[M_{t}(x, \alpha) \mid \mathscr{M}_{s}\right]=M_{s}(x, \alpha)$ for $t \geq s$.

Proof. We consider the case $(x, \alpha)=(0,0)$ for notational simplicity; the proof for $(x, \alpha) \neq(0,0)$ is similar. Let $\mathscr{S}_{\mathrm{NR}}([u, v])$ be $\mathscr{S}_{\mathrm{NR}}$ with the integration domain in $\int_{0}^{t} \cdots$ replaced by $\int_{u}^{v} \cdots$. Write $M_{t}=M_{t}(0,0)$. We see that

$$
\mathbb{E}_{P \times \mu}^{0,0}\left[M_{t} \mid \mathscr{M}_{s}\right]=e^{t(E+1)} e^{\mathscr{S}_{\mathrm{NR}}([0, s])} \mathbb{E}_{P \times \mu}^{0,0}\left[e^{\mathscr{S}_{\mathrm{NR}}([s, t])} \varphi_{\mathrm{g}}\left(B_{t}, \theta_{t}\right) \mid \mathscr{M}_{s}\right] .
$$

By the Markov property of the $\mathbb{R}^{3} \times \mathbb{Z}_{2}$-valued stochastic process $\left(B_{t}, N_{t}\right)_{t \geq 0}$ we have

$$
\begin{aligned}
& \mathbb{E}_{P \times \mu}^{0,0}\left[e^{\mathscr{S}_{\mathrm{NR}}([s, t])} \varphi_{\mathrm{g}}\left(B_{t}, \theta_{t}\right) \mid \mathscr{M}_{s}\right] \\
= & \mathbb{E}_{P \times \mu}^{B_{s}, N_{s}}\left[e^{-\int_{0}^{t-s} V\left(B_{r}\right) d r} e^{-i \int_{0}^{t-s} a\left(B_{r}\right) \circ d B_{r}} e^{\int_{0}^{t-s} U_{\mathrm{d}}\left(B_{r}, \theta_{r}\right) d r} e^{K} \varphi_{\mathrm{g}}\left(B_{t-s}, \theta_{t-s}\right)\right] .
\end{aligned}
$$

The off-diagonal part $K$ in $(57)$ is

$$
\begin{aligned}
K & =\sum_{\substack{s<u \leq t \\
N_{(u-s)+\neq N_{(u-s)-}}}} \log \left(-U_{\text {od }}\left(B_{u-s},-\theta_{(u-s)-}\right)\right) \\
& =\sum_{\substack{0<r \leq t-s \\
N_{r+\neq} \neq N_{r-}}} \log \left(-U_{\text {od }}\left(B_{r},-\theta_{r-}\right)\right)=\int_{0}^{(t-s)+} \log \left(-U_{\text {od }}\left(B_{r},-\theta_{r-}\right)\right) d N_{r} .
\end{aligned}
$$

Hence we conclude that

$$
\mathbb{E}_{P \times \mu}^{0,0}\left[e^{\mathscr{S}_{\mathrm{NR}}([s, t])} \varphi_{\mathrm{g}}\left(B_{t}, \theta_{t}\right) \mid \mathscr{M}_{s}\right]=\mathbb{E}_{P \times \mu}^{B_{s}, N_{s}}\left[e^{\mathscr{S}_{\mathrm{NR}}([0, t-s])} \varphi_{\mathrm{g}}\left(B_{t-s}, \theta_{t-s}\right)\right]
$$

which implies that

$$
\mathbb{E}_{P \times \mu}^{0,0}\left[M_{t} \mid \mathscr{M}_{s}\right]=e^{s(E+1)} e^{\mathscr{S}_{\mathrm{NR}}([0, s])} \mathbb{E}_{P \times \mu}^{B_{s}, N_{s}}\left[M_{t-s}\right]=M_{s} .
$$

Thus the lemma follows.

\section{§4.2. Martingale properties: relativistic case}

Next we discuss the relativistic case $H+V$. Let $\varphi_{\mathrm{g}}$ be a bound state of $H+V$ such that

$$
(H+V) \varphi_{\mathrm{g}}=E \varphi_{\mathrm{g}}
$$


for $E \in \mathbb{R}$. We use the same notation $\varphi_{\mathrm{g}}$ as for the non-relativistic case. Consider the stochastic process

$$
Y_{t}=e^{t E} e^{T_{t}} e^{\mathscr{S}} \varphi_{\mathrm{g}}\left(q_{t}\right), \quad t \geq 0
$$

Furthermore, we define

$$
Y_{t}(x, \alpha)=e^{t E} e^{T_{t}} e^{\mathscr{S}(x, \alpha)} \varphi_{\mathrm{g}}\left(q_{t}(x, \alpha)\right), \quad t \geq 0,
$$

where $q_{t}(x, \alpha)=\left(B_{T_{t}}+x,(-1)^{\alpha} \theta_{T_{t}}\right)$ and $\mathscr{S}(x, \alpha)=\mathscr{S}_{V}(x)+\mathscr{S}_{\mathrm{A}}(x)+\mathscr{S}_{\mathrm{S}}(x, \alpha)$ is given by

$$
\begin{aligned}
\mathscr{S}_{V}(x)= & -\int_{0}^{t} V\left(B_{T_{s}}+x\right) d s \\
\mathscr{S}_{\mathrm{A}}(x)= & -i \int_{0}^{T_{t}} a\left(B_{s}+x\right) \circ d B_{s}, \\
\mathscr{S}_{\mathrm{S}}(x, \alpha)= & -\int_{0}^{T_{t}} U_{\mathrm{d}}\left(B_{s}+x,(-1)^{\alpha} \theta_{s}\right) d s \\
& +\int_{0}^{T_{t}+} \log \left(-U_{\mathrm{od}}\left(B_{s}+x,-(-1)^{\alpha} \theta_{s-}\right)\right) d N_{s} .
\end{aligned}
$$

Then

$$
\mathbb{E}_{M}^{x, \alpha, 0}\left[Y_{t}\right]=\mathbb{E}_{M}^{0,0,0}\left[Y_{t}(x, \alpha)\right]=\varphi_{\mathrm{g}}\left(x,(-1)^{\alpha}\right) .
$$

We introduce a filtration under which $\left(Y_{t}\right)_{t \geq 0}$ is a martingale. Define $Y_{t}(\omega)$ and $Y_{t}(x, \alpha, \omega)$ for every $\omega \in \Omega_{\nu}$ to be $Y_{t}$ and $Y_{t}(x, \alpha)$, respectively, with subordinator $T_{t}$ replaced by the number $T_{t}(\omega) \geq 0$. Let

$$
\mathscr{F}_{t}^{(1)}(\omega)=\sigma\left(\left(B_{r}, N_{r}\right), 0 \leq r \leq T_{t}(\omega)\right) \in \mathscr{F}_{P} \times \mathscr{F}_{\mu}
$$

for $\omega \in \Omega_{\nu}$, and define

$$
\begin{aligned}
& \mathscr{F}_{t}^{(1)}=\left\{\bigcup_{\omega \in \Omega_{\nu}}(A(\omega), \omega) \mid A(\omega) \in \mathscr{F}_{t}^{(1)}(\omega)\right\} \subset \mathscr{F}_{P} \times \mathscr{F}_{\mu} \times \mathscr{F}_{\nu}, \\
& \mathscr{F}_{t}^{(2)}=\left\{\bigcup_{\omega \in \Omega_{P} \times \Omega_{N}}(\omega, B(\omega)) \mid B(\omega) \in \sigma\left(T_{r}, 0 \leq r \leq t\right)\right\} \subset \mathscr{F}_{P} \times \mathscr{F}_{\mu} \times \mathscr{F}_{\nu} .
\end{aligned}
$$

We see that $\mathscr{F}_{t}^{(1)}$ and $\mathscr{F}_{t}^{(2)}$ are sub- $\sigma$-fields of $\mathscr{F}_{P} \times \mathscr{F}_{\mu} \times \mathscr{F}_{\nu}$. Write

$$
\mathscr{F}_{t}=\mathscr{F}_{t}^{(1)} \cap \mathscr{F}_{t}^{(2)}, \quad t \geq 0 .
$$

The conditional expectation $\mathbb{E}_{M}^{0,0,0}\left[Y_{t}(x, \alpha) \mid \mathscr{F}_{t}^{(1)}\right]=\mathbb{E}_{M}^{0,0,0}\left[Y_{t}(x, \alpha) \mid \mathscr{F}_{t}^{(1)}\right](\cdot, \cdot, \cdot)$ is a stochastic process on $\Omega_{P} \times \Omega_{N} \times \Omega_{\nu}$. 
Lemma 4.2. We have

$$
\mathbb{E}_{M}^{0,0,0}\left[Y_{t}(x, \alpha) \mid \mathscr{F}_{t}^{(1)}\right](\cdot, \cdot, \omega)=\mathbb{E}_{P \times \mu}^{0,0}\left[Y_{t}(x, \alpha, \omega) \mid \mathscr{F}_{t}^{(1)}(\omega)\right](\cdot, \cdot)
$$

for all $\omega \in \Omega_{\nu}$.

Proof. Let $A=\bigcup_{\omega \in \Omega_{\nu}}(A(\omega), \omega)$ with $A(\omega) \in \mathscr{F}_{t}^{(1)}(\omega)$. Then

$$
\begin{aligned}
\mathbb{E}_{M}^{0,0,0}\left[1_{A} Y_{t}(x, \alpha)\right] & =\int_{\Omega_{\nu}} d \nu(\omega) \mathbb{E}_{P \times \mu}^{0,0}\left[1_{A(\omega)} Y_{t}(x, \alpha, \omega)\right] \\
& =\int_{\Omega_{\nu}} d \nu(\omega) \mathbb{E}_{P \times \mu}^{0,0}\left[1_{A(\omega)}(\cdot, \cdot) \mathbb{E}_{P \times \mu}^{0,0}\left[Y_{t}(x, \alpha, \omega) \mid \mathscr{F}_{t}^{(1)}(\omega)\right](\cdot, \cdot)\right] .
\end{aligned}
$$

On the other hand, we have

$$
\begin{aligned}
\mathbb{E}_{M}^{0,0,0}\left[1_{A} Y_{t}(x, \alpha)\right] & =\mathbb{E}_{M}^{0,0,0}\left[1_{A} \mathbb{E}_{M}^{0,0,0}\left[Y_{t}(x, \alpha) \mid \mathscr{F}_{t}^{(1)}\right]\right] \\
& =\int_{\Omega_{\nu}} d \nu(\omega) \mathbb{E}_{P \times \mu}^{0,0}\left[1_{A(\omega)}(\cdot, \cdot) \mathbb{E}_{M}^{0,0,0}\left[Y_{t}(x, \alpha) \mid \mathscr{F}_{t}^{(1)}\right](\cdot, \cdot, \omega)\right]
\end{aligned}
$$

A comparison of the two formulas above completes the proof.

Lemma 4.3. The stochastic process $\left(Y_{t}(x, \alpha)\right)_{t \geq 0}$ is a martingale with respect to $\left(\mathscr{F}_{t}\right)_{t \geq 0}$, i.e., $\mathbb{E}_{M}^{0,0,0}\left[Y_{t}(x, \alpha) \mid \mathscr{F}_{s}\right]=Y_{s}(x, \alpha)$ for $t \geq s$.

Proof. We consider the case $(x, \alpha)=(0,0)$ to keep the notation simple; the proof for $(x, \alpha) \neq(0,0)$ is again similar.

Note that

$$
\mathbb{E}_{M}^{0,0,0}\left[Y_{t} \mid \mathscr{F}_{s}\right]=\mathbb{E}_{M}^{0,0,0}\left[Y_{t} \mid \mathscr{F}_{s}^{(1)} \cap \mathscr{F}_{s}^{(2)}\right]=\mathbb{E}_{M}^{0,0,0}\left[\mathbb{E}_{M}^{0,0,0}\left[Y_{t} \mid \mathscr{F}_{s}^{(1)}\right] \mid \mathscr{F}_{s}^{(2)}\right]
$$

We first compute $\mathbb{E}_{P \times \mu}^{0,0}\left[Y_{t}(\omega) \mid \mathscr{F}_{s}^{(1)}(\omega)\right]$. Write

$$
\begin{aligned}
\mathscr{S}([u, v])= & -\int_{u}^{v} V\left(B_{T_{r}}\right) d r-i \int_{T_{u}}^{T_{v}} a\left(B_{r}\right) \circ d B_{r} \\
& -\int_{T_{u}}^{T_{v}} U_{\mathrm{d}}\left(B_{r}, \theta_{r}\right) d r+\int_{T_{u}}^{T_{v}+} \log \left(-U_{\text {od }}\left(B_{r},-\theta_{r-}\right)\right) d N_{r}
\end{aligned}
$$

and, for every $\omega \in \Omega_{\nu}$,

$$
\begin{aligned}
\mathscr{S}([u, v], \omega)= & -\int_{u}^{v} V\left(B_{T_{r}(\omega)}\right) d r-i \int_{T_{u}(\omega)}^{T_{v}(\omega)} a\left(B_{r}\right) \circ d B_{r} \\
& -\int_{T_{u}(\omega)}^{T_{v}(\omega)} U_{\mathrm{d}}\left(B_{r}, \theta_{r}\right) d r+\int_{T_{u}(\omega)}^{T_{v}(\omega)+} \log \left(-U_{\mathrm{od}}\left(B_{r},-\theta_{r-}\right)\right) d N_{r}
\end{aligned}
$$


and $q_{t}(\omega)=\left(B_{T_{t}(\omega)}, \theta_{T_{t}(\omega)}\right), t \geq 0$. Since $T_{t}(\omega)$ is non-random, we see in a similar way to the non-relativistic case that

$$
\begin{aligned}
& \mathbb{E}_{P \times \mu}^{0,0}\left[Y_{t}(\omega) \mid \mathscr{F}_{s}^{(1)}(\omega)\right] \\
& =e^{t E} e^{T_{t}(\omega)} e^{\mathscr{S}([0, s], \omega)} \mathbb{E}_{P \times \mu}^{0,0}\left[e^{\mathscr{S}([s, t], \omega)} \varphi_{\mathrm{g}}\left(q_{t}(\omega)\right) \mid \mathscr{F}_{s}^{(1)}(\omega)\right] \\
& =e^{t E} e^{T_{t}(\omega)} e^{\mathscr{S}([0, s], \omega)} \mathbb{E}_{P \times \mu}^{B_{T_{s}(\omega)}, N_{T_{s}(\omega)}}\left[e^{-\int_{s}^{t} V\left(B_{T_{r}(\omega)-T_{s}(\omega)}\right) d r} e^{-i \int_{T_{s}(\omega)}^{T_{t}(\omega)} a\left(B_{r-T_{s}}(\omega)\right) \circ d B_{r}}\right. \\
& \times e^{-\int_{T_{S}(\omega)}^{T_{t}(\omega)} U_{\mathrm{d}}\left(B_{r-T_{S}(\omega)}, \theta_{r-T_{S}(\omega)}\right) d r} e^{\int_{T_{S}(\omega)}^{T_{t}(\omega)+} \log \left(-U_{\mathrm{od}}\left(B_{r-T_{S}(\omega)},-\theta_{\left(r-T_{S}(\omega)\right)-}\right) d N_{r}\right.} \\
& \left.\times \varphi_{\mathrm{g}}\left(B_{T_{t}(\omega)-T_{s}(\omega)}, \theta_{T_{t}(\omega)-T_{s}(\omega)}\right)\right] .
\end{aligned}
$$

Hence by Lemma 4.2 we have

$$
\mathbb{E}_{M}^{0,0,0}\left[Y_{t} \mid \mathscr{F}_{s}^{(1)}\right]=e^{t E} e^{T_{s}} e^{\mathscr{S}([0, s])} Z_{t, s}
$$

where

$$
\begin{aligned}
& Z_{t, s}=e^{T_{t}-T_{s}} \mathbb{E}_{P \times \mu}^{B_{T_{s}}, N_{T_{s}}}\left[e^{-\int_{s}^{t} V\left(B_{T_{r}-T_{s}}\right) d r} e^{-i \int_{T_{s}}^{T_{t}} a\left(B_{r-T_{s}}\right) \circ d B_{r}}\right. \\
& \left.\quad \times e^{-\int_{T_{s}}^{T_{t}} U_{\mathrm{d}}\left(B_{r-T_{s}}, \theta_{r-T_{s}}\right) d r} e^{\int_{T_{s}}^{T_{t}+} \log \left(-U_{\mathrm{od}}\left(B_{r-T_{s}},-\theta_{\left(r-T_{s}\right)-}\right)\right) d N_{r}} \varphi_{\mathrm{g}}\left(B_{T_{t}-T_{s}}, \theta_{T_{t}-T_{s}}\right)\right] .
\end{aligned}
$$

Here $Z_{t, s}$ is given by

$$
\begin{aligned}
e^{u-v} \mathbb{E}_{P \times \mu}^{B_{u}, N_{v}} & {\left[e^{-\int_{s}^{t} V\left(B_{T_{r}-u}\right) d r} e^{-i \int_{v}^{u} a\left(B_{r-v}\right) \circ d B_{r}}\right.} \\
& \left.\times e^{-\int_{v}^{u} U_{\mathrm{d}}\left(B_{r-v}, \theta_{r-v}\right) d r} e^{\int_{v}^{u+} \log \left(-U_{\text {od }}\left(B_{r-v},-\theta_{(r-v)-}\right) d N_{r}\right.} \varphi_{\mathrm{g}}\left(B_{u-v}, \theta_{u-v}\right)\right]
\end{aligned}
$$

evaluated at $u=T_{t}$ and $v=T_{s}$. Take the conditional expectation of the right hand side above with respect to $\mathscr{F}_{s}^{(2)}$. We note that

$$
\mathbb{E}_{M}^{0,0,0}\left[f \mid \mathscr{F}_{s}^{(2)}\right]\left(\omega_{1}, \omega_{2}, \cdot\right)=\mathbb{E}_{\nu}^{0}\left[f\left(\omega_{1}, \omega_{2}, \cdot\right) \mid \mathscr{N}_{s}\right](\cdot),
$$

where $\mathscr{N}_{s}=\sigma\left(T_{r}, 0 \leq r \leq s\right)$. Since $e^{t E} e^{T_{s}} e^{\mathscr{S}([0, s])}$ is measurable with respect to $\mathscr{F}_{s}^{(2)}$, by (69) we consider the conditional expectation of $Z_{t, s}$ giving

$$
\begin{aligned}
& \mathbb{E}_{M}^{0,0,0}\left[Z_{t, s} \mid \mathscr{F}_{s}^{(2)}\right] \\
& =\mathbb{E}_{\nu}^{0}\left[e ^ { T _ { t } - T _ { s } } \mathbb { E } _ { P \times \mu } ^ { B _ { T _ { s } } , N _ { T _ { s } } } \left[e^{-\int_{s}^{t} V\left(B_{T_{r}-T_{s}}\right) d r} e^{-i \int_{0}^{T_{t}-T_{s}} a\left(B_{r}\right) \circ d B_{r}}\right.\right. \\
& \left.\left.\quad \times e^{-\int_{0}^{T_{t}-T_{s}} U_{\mathrm{d}}\left(B_{r}, \theta_{r}\right) d r} e^{\int_{0}^{\left(T_{t}-T_{s}\right)+} \log \left(-U_{\mathrm{od}}\left(B_{r},-\theta_{r-}\right)\right) d N_{r}} \varphi_{\mathrm{g}}\left(B_{T_{t}-T_{s}}, \theta_{T_{t}-T_{s}}\right)\right] \mid \mathscr{N}_{s}\right]
\end{aligned}
$$

where we used the Markov property of $\left(\left(B_{t}, N_{t}\right)\right)_{t>0}$. By the Markov property of $\left(T_{t}\right)_{t \geq 0}$ the above equals

$$
\begin{aligned}
& \mathbb{E}_{\nu}^{T_{s}}\left[e ^ { T _ { t - s } - T _ { 0 } } \mathbb { E } _ { P \times \mu } ^ { B _ { T _ { 0 } } , N _ { T _ { 0 } } } \left[e^{-\int_{s}^{t} V\left(B_{T_{r-s}-T_{0}}\right) d r} e^{-i \int_{0}^{T_{t-s}-T_{0}} a\left(B_{r}\right) \circ d B_{r}}\right.\right. \\
& \left.\left.\times e^{-\int_{0}^{T_{t-s}-T_{0}} U_{\mathrm{d}}\left(B_{r}, \theta_{r}\right) d r} e^{\int_{0}^{\left(T_{t-s}-T_{0}\right)+} \log \left(-U_{\mathrm{od}}\left(B_{r},-\theta_{r-}\right)\right) d N_{r}} \varphi_{\mathrm{g}}\left(B_{T_{t-s}-T_{0}}, \theta_{T_{t-s}-T_{0}}\right)\right]\right] .
\end{aligned}
$$


Since $\mathbb{E}_{\nu}^{u}[f(T)]=.\mathbb{E}_{\nu}^{0}[f(T .+u)]$, we see that this equals

$$
\begin{gathered}
\mathbb{E}_{\nu}^{0}\left[e ^ { T _ { t - s } - T _ { 0 } } \mathbb { E } _ { P \times \mu } ^ { B _ { T _ { 0 } + u } , N _ { T _ { 0 } + u } } \left[e^{-\int_{s}^{t} V\left(B_{T_{r-s}}-T_{0}\right) d r} e^{-i \int_{0}^{T_{t-s}-T_{0}} a\left(B_{r}\right) \circ d B_{r}}\right.\right. \\
\times e^{-\int_{0}^{T_{t-s}-T_{0}} U_{\mathrm{d}}\left(B_{r}, \theta_{r}\right) d r} e^{\int_{0}^{\left(T_{t-s}-T_{0}\right)+} \log \left(-U_{\mathrm{od}}\left(B_{r},-\theta_{r-}\right)\right) d N_{r}} \\
\left.\left.\times \varphi_{\mathrm{g}}\left(B_{T_{t-s}-T_{0}}, \theta_{T_{t-s}-T_{0}}\right)\right]\right]\left.\right|_{u=T_{s}} \\
=\mathbb{E}_{M}^{B_{T_{s}}, N_{T_{s}}, 0}\left[e^{T_{t-s}} e^{-\int_{0}^{t-s} V\left(B_{T_{r}}\right) d r} e^{-i \int_{0}^{T_{t-s}} a\left(B_{r}\right) \circ d B_{r}}\right. \\
\left.\times e^{-\int_{0}^{T_{t-s}} U_{\mathrm{d}}\left(B_{r}, \theta_{r}\right) d r} e^{\int_{0}^{T_{t-s}+} \log \left(-U_{\mathrm{od}}\left(B_{r},-\theta_{r-}\right)\right) d N_{r}} \varphi_{\mathrm{g}}\left(q_{t-s}\right)\right] \\
=\left(e^{-(t-s) H_{\mathbb{Z}_{2}}} \varphi_{\mathrm{g}}\right)\left(q_{s}\right) .
\end{gathered}
$$

Hence we conclude that

$$
\mathbb{E}_{M}^{0,0,0}\left[Y_{t} \mid \mathscr{F}_{s}\right]=e^{s E} e^{T_{s}} e^{\mathscr{S}([0, s])}\left(e^{-(t-s)\left(H_{\mathbb{Z}_{2}}-E\right)} \varphi_{\mathrm{g}}\right)\left(q_{s}\right)=Y_{s},
$$

and the lemma follows.

\section{§4.3. Upper estimates on bound states}

We will use the following conditions.

Assumption 4.4. (1) $b_{3} \in L^{\infty}$ and $W=\sqrt{b_{1}^{2}+b_{2}^{2}} \in L^{\infty}$.

(2) With $m_{*}=\left\|b_{3}\right\|_{\infty}+\|W\|_{\infty}$, we have $m_{*}<m^{2} / 2$.

(3) $V$ is of relativistic Kato class, i.e.,

$$
\lim _{t \downarrow 0} \sup _{x \in \mathbb{R}^{3}} \mathbb{E}_{P \times \nu}^{x, 0}\left[\int_{0}^{t} V\left(B_{T_{r}}\right) d r\right]=0 .
$$

Lemma 4.5. If Assumption 4.4 holds, then $\varphi_{\mathrm{g}} \in L^{\infty}\left(\mathbb{R}^{3}\right)$ and

$$
\left|\varphi_{\mathrm{g}}\left(x,(-1)^{\alpha}\right)\right| \leq \mathbb{E}_{P \times \nu}^{0,0}\left[e^{(t \wedge \tau) E} e^{-\int_{0}^{t \wedge \tau} V\left(B_{T_{r}}+x\right) d r} e^{\frac{1}{2} T_{t \wedge \tau} m_{*}}\right]\left\|\varphi_{\mathrm{g}}\right\|
$$

for every stopping time $\tau$ with respect to $\left(\mathscr{F}_{s}\right)_{s \geq 0}$ and $t \geq 0$.

Proof. Notice that $\varphi_{\mathrm{g}}\left(x,(-1)^{\alpha}\right)=\mathbb{E}_{M}^{x, \alpha, 0}\left[Y_{t}\right]$ for every $t$. Then the Schwarz inequality yields that

$$
\begin{aligned}
\left|\varphi_{\mathrm{g}}\left(x,(-1)^{\alpha}\right)\right| \leq & e^{t E} \mathbb{E}_{M}^{x, \alpha, 0}\left[e^{2 T_{t}} e^{-2 \int_{0}^{t} V\left(B_{T_{r}}\right) d r} e^{\int_{0}^{T_{t}}\left|b_{3}\left(B_{r}\right)\right| d r} e^{\int_{0}^{T_{t}+} \log W\left(B_{r}\right) d N_{r}}\right]^{1 / 2} \\
& \times \mathbb{E}_{M}^{x, \alpha, 0}\left[\left|\varphi_{\mathrm{g}}\left(q_{t}\right)\right|\right]^{1 / 2} \\
\leq & e^{t E}\left(\mathbb { E } _ { P \times \nu } ^ { 0 , 0 } \left[e^{-2 \int_{0}^{t} V\left(B_{T_{r}}+x\right) d r} e^{\left.\left.T_{t} m_{*}\right]\right)^{1 / 2}\left(\mathbb{E}_{M}^{x, \alpha, 0}\left[\left|\varphi_{\mathrm{g}}\left(q_{t}\right)\right|^{2}\right]\right)^{1 / 2} .}\right.\right.
\end{aligned}
$$

Here we used that $\mathbb{E}_{\mu}^{0}\left[e^{N_{T_{t}} \log W}\right]=e^{T_{t}(W-1)}$. Note that

$$
\mathbb{E}_{M}^{x, \alpha, 0}\left[\left|\varphi_{\mathrm{g}}\left(q_{t}\right)\right|^{2}\right]=\int_{0}^{\infty} d s p_{t}(s) \int_{\mathbb{R}^{3}} \Pi_{s}(y) d y \sum_{n=0}^{\infty}\left|\varphi_{\mathrm{g}}\left(x+y,(-1)^{\alpha+n}\right)\right|^{2} \frac{s^{n}}{n !} e^{-s},
$$


where

$$
p_{t}(s)=\frac{t e^{t m}}{\sqrt{2 \pi s^{3}}} e^{-\frac{1}{2}\left(t^{2} / s+m^{2} s\right)} 1_{[0, \infty)}(s)
$$

denotes the distribution of the subordinator $T_{t}$. Since

$$
\sum_{n=0}^{\infty}\left|\varphi_{\mathrm{g}}\left(x+y,(-1)^{\alpha+n}\right)\right|^{2} \frac{s^{n}}{n !} e^{-s} \leq\left|\varphi_{\mathrm{g}}(x+y, 1)\right|^{2}+\left|\varphi_{\mathrm{g}}(x+y,-1)\right|^{2},
$$

we obtain

$$
\begin{aligned}
\mathbb{E}_{M}^{x, \alpha, 0}\left[\left|\varphi_{\mathrm{g}}\left(q_{t}\right)\right|^{2}\right] & \leq \int_{\mathbb{R}^{3}} d y \int_{0}^{\infty} d s p_{t}(s) \Pi_{s}(y)\left(\left|\varphi_{\mathrm{g}}(x+y, 1)\right|^{2}+\left|\varphi_{\mathrm{g}}(x+y,-1)\right|^{2}\right) \\
& =\int_{\mathbb{R}^{3}} d y\left(\left|\varphi_{\mathrm{g}}(x+y, 1)\right|^{2}+\left|\varphi_{\mathrm{g}}(x+y,-1)\right|^{2}\right) \pi^{-2} \\
& \times \frac{t e^{m t}}{\left(|y|^{2}+t^{2}\right)^{2}} \int_{0}^{\infty} \xi e^{-\left(\xi+\frac{m^{2}\left(|y|^{2}+t^{2}\right) / 4}{\xi}\right)} d \xi \\
& \leq C_{t}\left\|\varphi_{\mathrm{g}}\right\|_{L^{2}\left(\mathbb{R}^{3} \times \mathbb{Z}_{2}\right)}^{2}
\end{aligned}
$$

with a constant $C_{t}$. Furthermore, let $m^{2} /\left(2 m_{*}\right)>q>1$ and $1 / p+1 / q=1$. Then

$$
\mathbb{E}_{P \times \nu}^{x, 0}\left[e^{-2 \int_{0}^{t} V\left(B_{T_{r}}\right) d r} e^{T_{t} m_{*}}\right] \leq\left(\mathbb{E}_{P \times \nu}^{x, 0}\left[e^{-2 p \int_{0}^{t} V\left(B_{T_{r}}\right) d r}\right]\right)^{1 / p}\left(\mathbb{E}_{P \times \nu}^{x, 0}\left[e^{q T_{t} m_{*}}\right]\right)^{1 / q} .
$$

The first term at the right hand side above satisfies

$$
\sup _{x \in \mathbb{R}^{3}}\left(\mathbb{E}_{P \times \nu}^{x, 0}\left[e^{-2 p \int_{0}^{t} V\left(B_{T_{r}}\right) d r}\right]\right)^{1 / p}<\infty,
$$

since $V$ is of relativistic Kato class, and

$$
\begin{aligned}
\mathbb{E}_{P \times \nu}^{x, 0}\left[e^{q T_{t} m_{*}}\right]=\mathbb{E}_{\nu}^{0}\left[e^{q T_{t} m_{*}}\right] & =\int_{0}^{\infty} e^{q s m_{*}} \frac{t e^{m t}}{\sqrt{2 \pi s^{3}}} e^{-\frac{1}{2}\left(t^{2} / s+m^{2} s\right)} d s \\
& =e^{+t\left(m-\sqrt{m^{2}-2 q m_{*}}\right)}<\infty
\end{aligned}
$$

Hence $\varphi_{\mathrm{g}} \in L^{\infty}\left(\mathbb{R}^{3}\right)$. Notice that by the martingale property of $Y_{t}(x, \alpha)$,

$$
\varphi_{\mathrm{g}}\left(x,(-1)^{\alpha}\right)=\mathbb{E}_{M}^{0,0,0}\left[Y_{t \wedge \tau}(x, \alpha)\right]
$$

for every stopping time $\tau$ and $t \geq 0$. Now (71) follows from (74) and

$$
\left|\varphi_{\mathrm{g}}\left(x,(-1)^{\alpha}\right)\right| \leq \mathbb{E}_{P \times \nu}^{0,0}\left[e^{(t \wedge \tau) E} e^{-\int_{0}^{t \wedge \tau} V\left(B_{T_{r}}+x\right) d r} e^{T_{t \wedge \tau} m_{*} / 2}\right]\left\|\varphi_{\mathrm{g}}\right\| .
$$

§4.4. Decay of bound states: the case $V \rightarrow \infty$

In this subsection we show the spatial exponential decay of bound states of $H+V$ at infinity. 
Lemma 4.6. Let $\tau_{R}=\inf \left\{t|| B_{T_{t}} \mid>R\right\}$. Then $\tau_{R}$ is a stopping time with respect to the filtration $\left(\mathscr{F}_{t}\right)_{t \geq 0}$.

Proof. It suffices to show that $\left\{\tau_{R} \leq t\right\} \in \mathscr{F}_{t}$. Notice that

$$
\left\{\tau_{R} \leq t\right\}=\bigcup_{\omega \in \Omega_{\nu}}(A(\omega), \omega)
$$

where $A(\omega)=\left\{\omega^{\prime} \in \Omega_{P}\left|\sup _{0 \leq s \leq t}\right| B_{T_{s}(\omega)}\left(\omega^{\prime}\right) \mid>R\right\} \in \mathscr{F}_{t}^{(1)}(\omega)$. Consequently, $\left\{\tau_{R} \leq t\right\} \in \mathscr{F}_{t}^{(1)}$. Moreover

$$
\left\{\tau_{R} \leq t\right\}=\bigcup_{\omega \in \Omega_{P}}(\omega, B(\omega))
$$

where $B(\omega)=\left\{\omega^{\prime} \in \Omega_{\nu}\left|\sup _{0 \leq s \leq t}\right| B_{T_{s}\left(\omega^{\prime}\right)}(\omega) \mid>R\right\}$. Therefore $\left\{\tau_{R} \leq t\right\} \in \mathscr{F}_{t}^{(2)}$ and hence $\left\{\tau_{R} \leq t\right\} \in \mathscr{F}_{t}$.

Theorem 4.7. If Assumption 4.4 holds and

$$
\lim _{|x| \rightarrow \infty} V(x)=\infty
$$

then for every $a>0$ there exists $b>0$ such that

$$
\left|\varphi_{\mathrm{g}}\left(x,(-1)^{\alpha}\right)\right| \leq b e^{-a|x|} .
$$

Proof. By Lemma 4.5 we have

$\left|\varphi_{\mathrm{g}}\left(x,(-1)^{\alpha}\right)\right|$

$$
\leq\left(\mathbb{E}_{P \times \nu}^{0,0}\left[e^{2\left(t \wedge \tau_{R}\right) E} e^{-2 \int_{0}^{t \wedge \tau_{R}} V\left(B_{T_{r}}+x\right) d r}\right]\right)^{1 / 2}\left(\mathbb{E}_{P \times \nu}^{0,0}\left[e^{m_{*} T_{t \wedge \tau}}\right]\right)^{1 / 2}\left\|\varphi_{\mathrm{g}}\right\| .
$$

Let $W(x)=W_{R}(x)=\inf \{V(y)|| x-y \mid<R\}$, and notice that

$$
\lim _{|x| \rightarrow \infty} W(x)-E=\infty .
$$

In particular, we may assume that $W(x)-E>0$. This gives

$$
\begin{aligned}
& \left(\mathbb{E}_{P \times \nu}^{0,0}\left[e^{2\left(t \wedge \tau_{R}\right) E} e^{-2 \int_{0}^{t \wedge \tau_{R}} V\left(B_{T_{r}}+x\right) d r}\right]\right)^{1 / 2} \\
& \leq\left(\mathbb{E}_{P \times \nu}^{0,0}\left[e^{-2\left(t \wedge \tau_{R}\right)(W(x)-E)}\right]\right)^{1 / 2} \\
& \leq\left(\mathbb{E}_{P \times \nu}^{0,0}\left[1_{\left\{\tau_{R}<t\right\}} e^{-2\left(t \wedge \tau_{R}\right)(W(x)-E)}\right]\right)^{1 / 2}+\left(\mathbb{E}_{P \times \nu}^{0,0}\left[1_{\left\{\tau_{R} \geq t\right\}} e^{-2\left(t \wedge \tau_{R}\right)(W(x)-E)}\right]\right)^{1 / 2} \\
& \leq\left(\mathbb{E}_{P \times \nu}^{0,0}\left[1_{\left\{\tau_{R}<t\right\}}\right]\right)^{1 / 2}+e^{-t(W(x)-E)} .
\end{aligned}
$$

We see that

$$
\begin{aligned}
\mathbb{E}_{P \times \nu}^{0,0}\left[1_{\left\{\tau_{R}<t\right\}}\right] & =\mathbb{E}_{P \times \nu}^{0,0}\left[1_{\left\{\sup _{0 \leq s \leq t}\left|B_{T_{s}}\right|-R \geq 0\right\}}\right] \\
& \leq \mathbb{E}_{P \times \nu}^{0,0}\left[e^{\alpha\left(\sup _{0 \leq s \leq t}\left|B_{T_{s}}\right|-R\right)}\right]
\end{aligned}
$$


for any $\alpha \geq 0$. It can be shown that $\left(\mathbb{E}_{P \times \nu}^{0,0}\left[e^{\alpha \sup _{0 \leq s \leq t}\left|B_{T_{s}}\right|}\right]\right)^{1 / 2} \leq C_{1} e^{C_{2} t}$ for sufficiently small $\alpha$ (see [CMS90, Proposition II.5]). Hence

$$
\left(\mathbb{E}_{P \times \nu}^{0,0}\left[1_{\left\{\tau_{R}<t\right\}}\right]\right)^{1 / 2} \leq e^{-\alpha R / 2} C_{1} e^{C_{2} t},
$$

and

$$
\left(\mathbb{E}_{P \times \nu}^{0,0}\left[e^{2\left(t \wedge \tau_{R}\right) E} e^{-2 \int_{0}^{t \wedge \tau_{R}} V\left(B_{T_{r}}+x\right) d r}\right]\right)^{1 / 2} \leq e^{-t(W(x)-E)}+e^{-\alpha R / 2} C_{1} e^{C_{2} t} .
$$

We also see that

$$
\begin{aligned}
\mathbb{E}_{P \times \nu}^{0,0}\left[e^{m_{*} T_{t \wedge \tau_{R}}}\right] & \leq \mathbb{E}_{P \times \nu}^{0,0}\left[1_{\left\{t<\tau_{R}\right\}} e^{m_{*} T_{t \wedge \tau_{R}}}\right]+\mathbb{E}_{P \times \nu}^{0,0}\left[1_{\left\{t \geq \tau_{R}\right\}} e^{m_{*} T_{t \wedge \tau_{R}}}\right] \\
& \leq \mathbb{E}_{P \times \nu}^{0,0}\left[e^{m_{*} T_{t}}\right]+\mathbb{E}_{P \times \nu}^{0,0}\left[1_{\left\{t \geq \tau_{R}\right\}} e^{m_{*} T_{\tau_{R}}}\right] \leq 2 \mathbb{E}_{\nu}^{0}\left[e^{m_{*} T_{t}}\right]
\end{aligned}
$$

where we used that $T_{\tau_{R}} \leq T_{t}$ for $\tau_{R} \leq t$. Thus we have

$$
\mathbb{E}_{P \times \nu}^{0,0}\left[e^{m_{*} T_{t \wedge \tau_{R}}}\right]^{1 / 2} \leq \sqrt{2} e^{t\left(m-\sqrt{m^{2}-2 m_{*}}\right) / 2}
$$

by (73). Hence by (79) and (80),

$$
\left|\varphi_{\mathrm{g}}\left(x,(-1)^{\alpha}\right)\right| \leq \sqrt{2}\left(e^{-t(W(x)-E)}+e^{-\alpha R / 2} C_{1} e^{C_{2} t}\right) e^{t\left(m-\sqrt{m^{2}-2 m_{*}}\right) / 2}\left\|\varphi_{\mathrm{g}}\right\| .
$$

Notice that by inserting $R=p|x|$ with any $0<p<1, W(x)-E=W_{R}(x)-E=$ $W_{p|x|}(x)-E \rightarrow \infty$ as $|x| \rightarrow \infty$. Thus substituting $t=\delta|x|$ for sufficiently small $\delta>0$ and $R=p|x|$ with some $0<p<1$ in (81) yields the conclusion.

\section{$\S 4.5$. Decay of bound states: the case $V \rightarrow 0$}

In this subsection we consider the case of potentials decaying to zero as $|x| \rightarrow \infty$.

Theorem 4.8. Let Assumption 4.4 hold and suppose that

$$
\lim _{|x| \rightarrow \infty} V(x)=0 .
$$

Also, assume that

$$
m-\sqrt{m^{2}-2 m_{*}}<-2 E .
$$

Then there exist $a, b>0$ such that

$$
\left|\varphi_{\mathrm{g}}\left(x,(-1)^{\alpha}\right)\right| \leq b e^{-a|x|} .
$$

Proof. Define $\tau_{R}=\tau_{R}(x)=\inf \left\{t \geq 0|| B_{T_{t}}+x \mid \leq R\right\}$. Then $\tau_{R}$ is a stopping time, which can be seen in the same way as in Lemma 4.6. Thus

$$
\begin{aligned}
\mid \varphi_{\mathrm{g}}(x, & \left.(-1)^{\alpha}\right) \mid \\
& \leq\left(\mathbb{E}_{P \times \nu}^{0,0}\left[e^{2\left(t \wedge \tau_{R}\right) E} e^{-2 \int_{0}^{t \wedge \tau_{R}} V\left(B_{T_{r}}+x\right) d r}\right]\right)^{1 / 2}\left(\mathbb{E}_{P \times \nu}^{0,0}\left[e^{m_{*} T_{t \wedge \tau_{R}}}\right]\right)^{1 / 2}\left\|\varphi_{\mathrm{g}}\right\| \\
& =\left(\mathbb{E}_{P \times \nu}^{x, 0}\left[e^{2\left(t \wedge \tau_{R}(0)\right) E} e^{-2 \int_{0}^{t \wedge \tau_{R}(0)} V\left(B_{T_{r}}\right) d r}\right]\right)^{1 / 2}\left(\mathbb{E}_{P \times \nu}^{x, 0}\left[e^{m_{*} T_{t \wedge \tau_{R}}(0)}\right]\right)^{1 / 2}\left\|\varphi_{\mathrm{g}}\right\|
\end{aligned}
$$


We rewrite $\tau_{R}(0)$ as $\tau_{R}$. Let $\varepsilon>0$ be arbitrary. Then for sufficiently large $R$ it follows that $\sup _{|x|>R}|V(x)|<\varepsilon$ by (82), and we see that $\left|\int_{0}^{t \wedge \tau_{R}} V\left(B_{T_{r}}\right) d r\right| \leq$ $\left(t \wedge \tau_{R}\right) \varepsilon$. This gives

$$
\left|\varphi_{\mathrm{g}}\left(x,(-1)^{\alpha}\right)\right| \leq\left(\mathbb{E}_{P \times \nu}^{x, 0}\left[e^{2\left(t \wedge \tau_{R}\right)(E+\varepsilon)}\right]\right)^{1 / 2}\left(\mathbb{E}_{P \times \nu}^{x, 0}\left[e^{\left.m_{*} T_{t \wedge \tau_{R}}\right]}\right)^{1 / 2}\left\|\varphi_{\mathrm{g}}\right\| .\right.
$$

Thus

$$
\begin{aligned}
\mathbb{E}_{P \times \nu}^{x, 0}\left[e^{2\left(t \wedge \tau_{R}\right)(E+\varepsilon)}\right] & =\mathbb{E}_{P \times \nu}^{x, 0}\left[1_{\left\{t \leq \tau_{R}\right\}} e^{2 t(E+\varepsilon)}\right]+\mathbb{E}_{P \times \nu}^{x, 0}\left[1_{\left\{t>\tau_{R}\right\}} e^{2 \tau_{R}(E+\varepsilon)}\right] \\
& \leq e^{2 t(E+\varepsilon)}+C_{1} e^{-m_{\varepsilon}|x|}
\end{aligned}
$$

by making use of [CMS90, (II.29)(II.22) and (IV.3)] as above, where

$$
m_{\varepsilon}= \begin{cases}m & \text { if } 2|E|>m, \\ 2 \sqrt{m|E|-|E|^{2}} & \text { if } 2|E| \leq m .\end{cases}
$$

Also, notice that

$$
\mathbb{E}_{P \times \nu}^{x, 0}\left[e^{m_{*} T_{t \wedge \tau_{R}}}\right] \leq 2 e^{t\left(m-\sqrt{m^{2}-2 m_{*}}\right)} .
$$

Therefore

$$
\left|\varphi_{\mathrm{g}}\left(x,(-1)^{\alpha}\right)\right| \leq\left(e^{t(E+\epsilon)}+C_{1} e^{-m_{\epsilon}|x| / 2}\right) \sqrt{2} e^{t\left(m-\sqrt{m^{2}-2 m_{*}}\right) / 2} .
$$

On inserting $t=\delta|x|$ with sufficiently small $\delta$, the theorem follows from (83).

\section{Acknowledgements}

FH acknowledges support of Grant-in-Aid for Science Research (B) 20340032 from JSPS and Grant-in-Aid for Challenging Exploratory Research 22654018 from JSPS, and thanks the hospitality of ICMS Edinburgh, where part of this work was done. JL also thanks ICMS Edinburgh for a Research-in-Group grant allowing him to complete the project of this paper. We thank Toshimitsu Takaesu for pointing out typing errors in the first version of the manuscript.

\section{References}

[ARS91] G. F. De Angelis, A. Rinaldi and M. Serva, Imaginary-time path integral for a relativistic spin-(1/2) particle in a magnetic field, Europhys. Lett. 14 (1991), 95-100. MR 1154808

[Car78] R. Carmona, Pointwise bounds for Schrödinger eigenstates, Comm. Math. Phys. 62 (1978), 97-106. Zbl 0403.47016 MR 0505706

[CMS90] R. Carmona, W. C. Masters and B. Simon, Relativistic Schrödinger operators: asymptotic behavior of the eigenfunctions, J. Funct. Anal. 91 (1990), 117-142. Zbl 0716.35006 MR 1054115

[HIL09] F. Hiroshima, T. Ichinose and J. Lőrinczi, Path integral representation for Schrödinger operator with Bernstein function of the Laplacian, Rev. Math. Phys. 24 (2012), 1250013. Zbl pre06082012 MR 2944475 
[HL08] F. Hiroshima and J. Lörinczi, Functional integral representation of the Pauli-Fierz model with spin 1/2, J. Funct. Anal. 254 (2008), 2127-2185. Zbl 1141.81020 MR 2402105

[LS81] H. Leinfelder and C. G. Simader, Schrödinger operators with singular magnetic potentials, Math. Z. 176 (1981), 1-19. Zbl 0468.35038 MR 0606167

[LHB09] J. Lőrinczi, F. Hiroshima and V. Betz, Feynman-Kac-type theorems and Gibbs measures on path space. With applications to rigorous quantum field theory, de Gruyter Stud. Math. 34, de Gruyter, 2011. Zbl 1236.81003 MR 2848339 